\title{
Straightforward continuous synthesis of $\alpha$-aminophosphonates under microreactor conditions
}

\author{
Ellen Van Meenen, Kristof Moonen, ${ }^{\S}$ Davy Acke, and Christian V. Stevens ${ }^{*}$ \\ Department of Organic Chemistry, Faculty of Bioscience Engineering, Ghent University, \\ Coupure Links 653, B-9000 Ghent, Belgium \\ E-mail: Chris.Stevens@UGent.be
}

\begin{abstract}
The synthesis of $\alpha$-aminophosphonates on a large scale is not always straightforward. One of the described methodologies has now been adjusted for use in a microreactor system. The CPC College System was used to investigate the continuous production of $\alpha$-aminophosphonates. This technology couples miniaturisation to optimal reaction conditions for the production of chemical and pharmaceutical intermediates. The reaction of an imine with a dialkyl phosphite in different solvents was optimised, since the use of a solvent is necessary when using a microreactor. When methanol was used, a considerable reduction of the reaction time was noticed in comparison with other commonly used solvents. Subsequently, several reaction parameters were optimised in batch and compared with the continuous process in the microreactor. Similar yields and purities were obtained compared to the batch reaction. Using the microreactor system, the scale-up problems were avoided and large amounts of $\alpha$ aminophosphonates could be generated in a short time.
\end{abstract}

Keywords: $\alpha$-Aminophosphonates, addition to imines, microreactor, continuous process

\section{Introduction}

$\alpha$-Aminophosphonates, structural analogues of the corresponding $\alpha$-amino acids, as well as heterocyclic phosphonates ${ }^{1}$ and $\omega$-aminophosphonates ${ }^{2}$, are considered as an important class of compounds, with several interesting biological activities. Because of their application as peptide mimetics, ${ }^{3}$ enzyme inhibitors, ${ }^{4}$ antibiotics and pharmacological agents, ${ }^{5}$ a great variety of synthetic methods has been developed. In order to be able to produce multigram quantities

\footnotetext{
$\S$ Aspirant of the Fund for Scientific Research - Flanders (FWO-Vlaanderen)

* Corresponding author. Tel:+32-9-264 5957 ; Fax:+32-9-264 62 43; e-mail : Chris.Stevens@UGent.be; URL: http://www.synbioc.ugent.be
} 
without coping with many scale-up difficulties, an appropriate and straightforward method compatible with the use of microreactor technology was investigated.

Microreactor technology tries to couple miniaturisation to optimal reaction conditions for the production of chemical and pharmaceutical intermediates. Several advantages are associated with the use of the microreactor, such as a better heat and mass transfer due to a greater surfaceto-volume ratio and better mixing of the products. ${ }^{6,7,8}$ This technology also allows switching from batch to continuous processing using similar reaction conditions without the need to scaleup. In this study, the CPC College System was evaluated (Figure 1). ${ }^{9}$

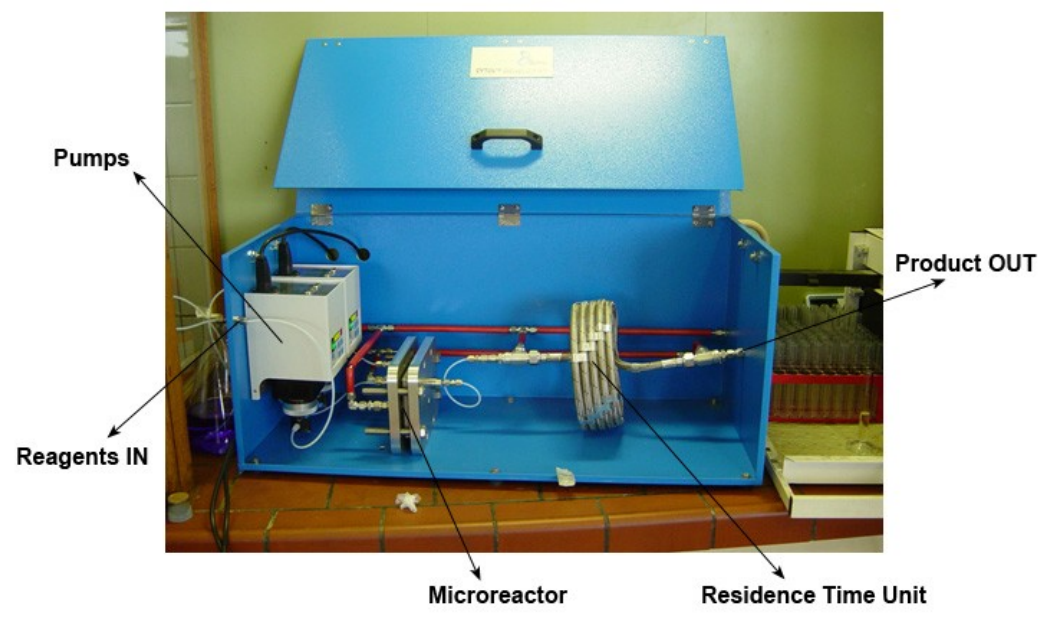

Figure 1. CPC College System - microreactor $^{9}$

Among the synthetic routes towards $\alpha$-aminophosphonates two main pathways exist: a) three-component reactions (also known as the Kabachnik-Fields reaction), in which an aldehyde, an amine and a di- or trialkyl phosphite are reacted in a one-pot set-up. A lot of research has been performed concerning the mechanism of this three component reaction. ${ }^{10,11}$ In some reports this straightforward one-pot-procedure is used without the use of any catalysts. ${ }^{12,13}$ However, in most cases this reaction is performed using catalysts, such as $\mathrm{LiClO}_{4},{ }^{14,15} \mathrm{TaCl}_{5}-\mathrm{SiO}_{2},{ }^{16} \mathrm{InCl}_{3}{ }^{17}$ $\mathrm{Sc}\left(\mathrm{O}_{3} \mathrm{SOC}_{12} \mathrm{H}_{25}\right)_{3},{ }^{18} \mathrm{SiO}_{2},{ }^{19}$ lanthanide-triflate ${ }^{20}$ and $\mathrm{CF}_{3} \mathrm{CO}_{2} \mathrm{H}^{21}$ The disadvantages of this methodology are often the moderate yields and sometimes the limited scope of the reaction; b) The second pathway is the Pudovik reaction, where dialkyl phosphites are added to compounds containing an imino-bond. Mechanistic studies have been performed, ${ }^{22,23,24}$ and many varieties of this reaction exist using either base ${ }^{25,26,27}$ or Lewis acid catalysis. Lewis acids that have been evaluated are $\mathrm{AlCl}_{3},{ }^{28} \mathrm{Me}_{2} \mathrm{AlCl}^{29}{ }^{29} \mathrm{BF}_{3},{ }^{30} \mathrm{SnCl}_{4}{ }^{31}$ and $\mathrm{ZrCl}_{4}{ }^{32}$ Other methods use silylated dialkyl phosphites, generated in situ, as a nucleophile. ${ }^{33,34,35,36}$ Dialkyl phosphite itself is generally considered as a poor nucleophile in its predominant $\sigma_{4} \lambda_{5}$ form, while O-silylation freezes out the $\sigma_{3} \lambda_{3}$ form. In some cases the addition of trialkyl phosphites, in stead of dialkyl phosphites, to imines ${ }^{37,38,39}$ or iminium salts ${ }^{40,41,42}$ is reported. 
A closer look into the literature, showed that all these catalysts are redundant. Fields already showed in 1952 that the reaction between an imine and dialkyl phosphites proceeds smoothly without catalysis and under solvent-free conditions. ${ }^{43,44}$ However, some kinetic experiments have been published recently, claiming the need of acid catalysis originating from a small degree of hydrolysis of the phosphite reagent. ${ }^{45}$

\section{Results and Discussion}

The aldimines used in this study were synthesised by treatment of the aldehyde with the primary amine in dichloromethane in the presence of anhydrous magnesium sulfate. After filtration and evaporation, the imines were isolated in almost quantitative yields. Fields' two component reaction is fast, high-yielding and moreover has a $100 \%$ atom efficiency. ${ }^{43}$ Since the microreactor has two inlets, this reaction seemed applicable to switch to a continuous process, except for the solvent-free conditions. Because of the high viscosities of the end products and the risk of crystallization (the end products were mainly crystalline), a solvent is needed to switch to a microreactor synthesis, in order to prevent clogging of the capillary tubes $(50 \mu \mathrm{m})$. Therefore, the initial step concerned the selection of an appropriate solvent in which the reaction proceeds rapidly since the maximum residence time of the microreactor utilised is approximately two hours. Complete conversion is required in this time in order to prevent laborious separation of the end product and reagents. The benzylidene(isopropyl)amine $\mathbf{1}$ was used for optimisation of the reaction conditions.

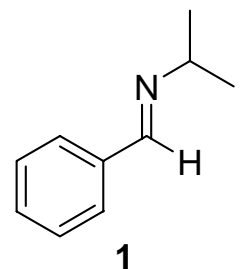

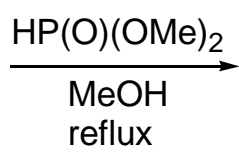

reflux

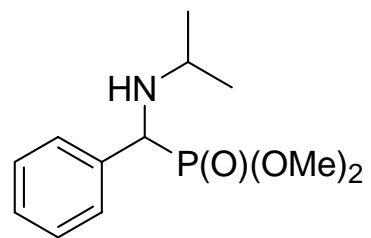

$2 a$

Scheme 1. Model reaction used for optimising the reaction conditions

In literature, the most common solvent utilised for this type of addition is dichloromethane. This solvent gave, however, unsatisfactory results as compared to the solvent-free conditions (Table 1). The reaction proceeded much slower and moreover, the reaction did not go to completion. When other solvents (acetonitrile, diethyl ether, methanol ${ }^{46}$ and toluene ${ }^{47}$ ) were screened, methanol seemed to give the fastest reaction, without formation of any side products. Diethyl ether gave almost no reaction and acetonitrile resulted in the formation of side products. Toluene provided reasonable results while $\mathrm{MeOH}$ obviously was the solvent of choice. Under batch conditions, four hours of reflux in methanol, which still is longer than the maximal residence time of the microreactor, still left $6 \%$ of starting imine in the reaction mixture. 
Table 1. Evaluation of solvents for use in the microreactor (1 equiv. phosphite) under batch conditions

\begin{tabular}{cccc}
\hline Entry & Solvent & $\begin{array}{c}\text { \% conversion } \\
\text { after } 1.5 \mathrm{~h}^{\mathrm{a}}\end{array}$ & $\begin{array}{c}\% \text { conversion } \\
\text { after } 22 \mathrm{~h}^{\mathrm{a}}\end{array}$ \\
\hline 1 & neat & 91 & - \\
2 & dichloromethane & 2 & 73 \\
3 & acetonitrile & 14 & $58^{\mathrm{b}}$ \\
4 & diethyl ether & 1 & 32 \\
5 & methanol & 90 & $94^{\mathrm{c}}$ \\
6 & toluene & 76 & 95 \\
\hline${ }^{\text {a }}$ determined by ${ }^{31} \mathrm{P}-\mathrm{NMR} ;{ }^{\mathrm{b}}$ formation of side products; ${ }^{\mathrm{c}}$ No change after $3.5 \mathrm{~h}$
\end{tabular}

Monitoring the reaction by ${ }^{31} \mathrm{P}-\mathrm{NMR}$, a dramatic drop of the reaction rate was noticed at the end of the reaction when reagents become limiting. Therefore, two equivalents of phosphite were used, decreasing the reaction time considerably to $80 \mathrm{~min}$ with $100 \%$ conversion, which was now below the residence time of the microreactor. Distilling the excess of phosphite from the reaction mixture was not successful, resulting in a partially breakdown of the product. Moreover, it proved to be hard to remove the excess phosphite completely. Finally, an acidbase-extraction after evaporation of the methanol in vacuo resulted in the total removal of the phosphite and the aminophosphonate 2a was recovered in high yield (94\%).

Subsequently, the method was extended by varying the amine, the aldehyde as well as the phosphite reagent (Table 2). Dimethyl and diethyl phosphite resulted in comparable yields, however, diisopropyl phosphite gave a much lower yield. While in the literature aromatic aldehydes were evaluated predominantly, in this report aliphatic aldehydes were also evaluated (entries 12-16). The results show clearly that this method is applicable on both aromatic and aliphatic aldimines. When $\alpha, \beta$-unsaturated imines were used (entries 16-21), no 1,4-addition was observed, which is in contrast with the addition of silylated dialkylphosphite. ${ }^{34}$

In a following experiment, the methanol and dimethyl phosphite were purified prior to use in order to establish the need of acid catalysis, originating from a small degree of hydrolysis of the phosphite reagent. ${ }^{45}$ Therefore, sodium metal was added to the reagents prior to distillation. The yields and purities were comparable with the previous results (without purifying the reagents), which proves that there is no need for acid catalysis. 
Table 2. Batch synthesis of $\alpha$-aminophosphonates in $\mathrm{MeOH}$

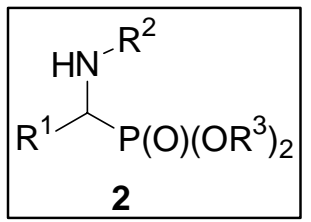

\begin{tabular}{|c|c|c|c|c|c|}
\hline Entry & product & $\mathrm{R}^{1}$ & $\mathrm{R}^{2}$ & $\mathrm{R}^{3}$ & Yield $(\%)$ \\
\hline 1 & $2 \mathbf{a}$ & $\mathrm{Ph}$ & $i-\operatorname{Pr}$ & $\mathrm{Me}$ & 94 \\
\hline 2 & $2 \mathbf{b}$ & $\mathrm{Ph}$ & $i-\operatorname{Pr}$ & $\mathrm{Et}$ & 84 \\
\hline 3 & $2 c$ & $\mathrm{Ph}$ & $i-\operatorname{Pr}$ & $i-\operatorname{Pr}$ & 45 \\
\hline 4 & $2 d$ & $\mathrm{Ph}$ & $t-\mathrm{Bu}$ & $\mathrm{Me}$ & 91 \\
\hline 5 & $2 \mathbf{e}$ & $\mathrm{Ph}$ & $\mathrm{Bn}$ & $\mathrm{Me}$ & 89 \\
\hline 6 & $2 f$ & $p-\mathrm{Cl}-\mathrm{Ph}$ & $i-\operatorname{Pr}$ & $\mathrm{Me}$ & 89 \\
\hline 7 & $2 g$ & $p$-Me-Ph & $i-\operatorname{Pr}$ & $\mathrm{Me}$ & 97 \\
\hline 8 & $2 h$ & $p-\mathrm{NO}_{2}-\mathrm{Ph}$ & $i-\operatorname{Pr}$ & $\mathrm{Me}$ & 88 \\
\hline 9 & $2 \mathbf{i}$ & 2-furyl & allyl & $\mathrm{Me}$ & 89 \\
\hline 10 & $2 \mathbf{j}$ & 2-furyl & $\mathrm{Bn}$ & $\mathrm{Me}$ & 89 \\
\hline 11 & $2 \mathbf{k}$ & 2-indolyl & $i-\operatorname{Pr}$ & $\mathrm{Me}$ & 80 \\
\hline 12 & 21 & $c$-Hex & $i-\operatorname{Pr}$ & $\mathrm{Me}$ & 93 \\
\hline 13 & $2 \mathrm{~m}$ & $i-\operatorname{Pr}$ & $i-\operatorname{Pr}$ & $\mathrm{Me}$ & 86 \\
\hline 14 & $2 n$ & $i-\operatorname{Pr}$ & $t-\mathrm{Bu}$ & $\mathrm{Me}$ & 83 \\
\hline 15 & 20 & $i-\operatorname{Pr}$ & $\mathrm{Bn}$ & $\mathrm{Me}$ & 84 \\
\hline 16 & $2 p$ & 2-methyl-1-propenyl & $i-\operatorname{Pr}$ & $\mathrm{Et}$ & 84 \\
\hline 17 & $2 q$ & 2-phenylethenyl & allyl & $\mathrm{Me}$ & 95 \\
\hline 18 & $2 r$ & 2-phenylethenyl & $t-\mathrm{Bu}$ & $\mathrm{Me}$ & 97 \\
\hline 19 & $2 s$ & 2-phenylethenyl & $i-\operatorname{Pr}$ & $\mathrm{Me}$ & 74 \\
\hline 20 & $2 t$ & 2-phenylethenyl & $i-\operatorname{Pr}$ & $\mathrm{Et}$ & 95 \\
\hline 21 & $2 \mathbf{u}$ & 2-phenylethenyl & $\mathrm{Bn}$ & $\mathrm{Et}$ & 95 \\
\hline
\end{tabular}

The next step of the research comprised the evaluation of the reaction in the microreactor. Therefore, reaction parameters such as the residence time, the amount of phosphite, the temperature and the concentration have been varied. There are, however, some restrictions concerning the temperature and the concentration. The reaction temperature is limited by the boiling point of the solvent in order to avoid cavitation effects in the reactor which gives undeterminable residence times and uncontrollable reaction conditions. The concentration on the 
other hand is limited in order to prevent crystallisation of the end product which could cause clogging of the capillary tubes $(50 \mu \mathrm{m})$.

First, the optimised batch conditions were tested (Table 3, entry 1), which resulted in a complete conversion and a comparable yield. Entries 2 to 4 show attempts to diminish the amount of phosphite. However, no complete conversion could be achieved.

Table 3. Optimization of the $\alpha$-aminophosphonate formation (2a) in the microreactor

\begin{tabular}{cccccccc}
\hline Entry & $\begin{array}{c}\text { Mass\% } \\
\text { in MR }\end{array}$ & $\begin{array}{c}\text { Residence } \\
\text { time }(\mathrm{min})\end{array}$ & Solvent & $\begin{array}{c}\mathrm{T} \\
\left({ }^{\circ} \mathrm{C}\right)\end{array}$ & $\begin{array}{c}\mathrm{HOP}(\mathrm{OMe})_{2} \\
\text { (equiv. })\end{array}$ & $\begin{array}{c}\text { Conversion } \\
(\mathrm{mol} \%)\end{array}$ & $\begin{array}{c}\text { Yield }^{\mathrm{a}} \\
(\%)\end{array}$ \\
\hline 1 & 10 & 78 & $\mathrm{MeOH}$ & 50 & 2 & 100 & 85 \\
2 & 10 & 87 & $\mathrm{MeOH}$ & 50 & 1.5 & 94 & 83 \\
3 & 10 & 87 & $\mathrm{MeOH}$ & 50 & 1.6 & 94 & 82 \\
4 & 10 & 87 & $\mathrm{MeOH}$ & 50 & 1.75 & 95 & 89 \\
$\mathbf{5}$ & $\mathbf{2 0}$ & $\mathbf{7 8}$ & $\mathbf{M e O H}$ & $\mathbf{5 0}$ & $\mathbf{2}$ & $\mathbf{1 0 0}$ & $\mathbf{8 2}$ \\
6 & 40 & 87 & $\mathrm{MeOH}$ & 50 & 2 & 67 & 24 \\
7 & 10 & 47 & $\mathrm{MeOH}$ & 50 & 2 & 93 & 83 \\
8 & 10 & 44 & $\mathrm{n}-\mathrm{BuOH}$ & 100 & 2 & 100 & $\mathrm{~b}$ \\
9 & 10 & 29 & $\mathrm{n}-\mathrm{BuOH}$ & 100 & 2 & 100 & $\mathrm{~b}$ \\
10 & 10 & 19 & $\mathrm{n}-\mathrm{BuOH}$ & 100 & 2 & 94 & $\mathrm{~b}$ \\
11 & 10 & 29 & $\mathrm{sec}-\mathrm{BuOH}$ & 85 & 2 & 100 & $\mathrm{~b}$ \\
\hline
\end{tabular}

${ }^{\mathrm{a}}$ Yield after acid-base extraction; ${ }^{\mathrm{b}}$ side products due to transesterification

When a higher concentration was applied in the reactor, a higher production rate of aminophosphonate could be achieved with less solvent consumption. However, increasing the concentration to $40 \%$ resulted in incomplete conversion, whereas a $20 \%$ concentration seemed to be optimal (entries 5-6). A possible explanation is that the higher viscosity diminishes the diffusion process which is an important feature in the small channels of the microreactor. Also the flow was doubled to study this influence (entry 7), but this resulted also in incomplete conversion.

Because of the low boiling point of methanol, it was difficult to increase the temperature. As an alternative, higher alcohols were tested as a solvent in order to evaluate the influence of the temperature (entries 8-11). With n-butanol as a solvent, the residence time could be diminished to 29 minutes with $100 \%$ conversion, but due to transesterification side products were formed. Sec-butanol was believed to reduce this transesterification due to a higher steric hindrance, but also in this case side products were formed. 
Again the generality of this method was investigated by varying the amine and the aldehyde. As can be seen in table 4, the continuous process allowed us to synthesize more than 10 grams $\alpha$-aminophosphonate per hour (or more then $250 \mathrm{~g}$ per day) without having the risk of handling big amounts of unpleasant chemicals in the lab. Several derivatives can be produced without alteration of the method, simply by switching of the aldimine.

Table 4. Continuous synthesis of different $\alpha$-aminophosphonates in the microreactor

\begin{tabular}{ccccccc}
\hline Entry & Product & $\mathrm{R}_{1}$ & $\mathrm{R}_{2}$ & $\mathrm{R}_{3}$ & Yield (\%) & Throughput $(\mathrm{g} / \mathrm{h})$ \\
\hline 1 & 2a & $\mathrm{Ph}$ & $i$-Pr & $\mathrm{Me}$ & 82 & 10.3 \\
2 & 2s & 2-phenylethenyl & $i$-Pr & $\mathrm{Me}$ & 91 & 10.7 \\
3 & 2l & $c$-Hex & $i$-Pr & $\mathrm{Me}$ & 78 & 9.7 \\
4 & 2o & i-Pr & $\mathrm{Bn}$ & $\mathrm{Me}$ & 68 & 8.2 \\
5 & 2i & 2-furyl & allyl & $\mathrm{Me}$ & 72 & 9.4 \\
\hline
\end{tabular}

Conditions: 20 mass \% imine, 2 eq. phosphite, residence time of $78 \mathrm{~min}$ (= flow rate of $0.6 \mathrm{~mL} / \mathrm{min}$ or $0.3 \mathrm{~mL} / \mathrm{min}$. pump)

In conclusion, we have developed a high-yielding, environmentally friendly continuous process for the synthesis of $\alpha$-aminophosphonates. Work-up consists of a simple acid-base extraction and results in pure end products avoiding laborious chromatographical purification. Furthermore, the reaction is also of interest in batch syntheses of $\alpha$-aminophosphonates. Given the simplicity and high yield of the condensation of imines with dialkyl phosphites, the large number of different methods described using a wide range of expensive additives and activation steps, is surprising.

\section{Experimental Section}

General Procedures. CPC ${ }^{\circledR}$ College System ${ }^{9}$ was used as the microreactor system. This system consists of a $2 \mathrm{~mL}$ microreactor and a $45 \mathrm{~mL}$ residence time unit. The pumps were calibrated at the desired flow rate. The temperature was controlled using an external circuit (Huber Tango thermostat). All reagents were used without prior purification before use (unless otherwise stated).

All reactions were monitored using thin-layer chromatography (TLC) carried out on $0.25-$ mm E. Merck silica gel plates (60F-254) using $\mathrm{KMnO}_{4}$.

NMR spectra were recorded on a JEOL Eclipse FT instrument using $\mathrm{CDCl}_{3}$ as solvent and TMS as internal reference ( ${ }^{1} \mathrm{H}-\mathrm{NMR}$ at $300 \mathrm{MHz}$ and ${ }^{13} \mathrm{C}-\mathrm{NMR}$ at $\left.75.4 \mathrm{MHz}\right) .{ }^{31} \mathrm{P}-\mathrm{NMR}$ spectra were recorded at $121.4 \mathrm{MHz}$ with $\mathrm{CDCl}_{3}$ as solvent. The following abbreviations are used to 
indicate the multiplicities: s, singlet; $d$, doublet; $t$, triplet; $q$, quartet; m, multiplet; br, broad signal. Low resolution mass spectra were obtained using an Agilent 1100 series VS via Electron Spray Ionisation geometry (positive mode or negative mode). High resolution mass spectra were recorded on a Finnigan MAT 95 XP-API-GC-Trap tandem Mass spectrometer system. IR spectra were recorded on a Perkin Elmer Spectrum One spectrophotometer and only typical absorptions were cited.

General procedure for the preparation of $\alpha$-aminophosphonates (2) in batch conditions. A mixture of aldimine $(5 \mathrm{mmol})$ and dialkyl phosphite $(10 \mathrm{mmol})$ in methanol was refluxed for one to three hours (depending on the type of the imine). After reaction, the solvent was evaporated in vacuo and the residue was dissolved in $10 \mathrm{~mL}$ of dichloromethane and extracted with $10 \mathrm{~mL}$ of $1 \mathrm{~N} \mathrm{HCl}$. The aqueous phase was washed 2 times with $10 \mathrm{~mL}$ of dichloromethane. Afterwards, the aqueous phase was basified using $1 \mathrm{~N} \mathrm{NaOH}$ and extracted 3 times with $10 \mathrm{~mL}$ of dichloromethane. The combined organic fractions were dried over $\mathrm{MgSO}_{4}$. After filtration and evaporation the pure $\alpha$-aminophosphonates 2 were obtained with yields between 74 and $97 \%$ in high purity.

General procedure for the preparation of $\alpha$-aminophosphonates (2) using the CPCmicroreactor. In the optimized procedure, a solution of 40 mass \% of the corresponding imine in $\mathrm{MeOH}$ was prepared and transferred in a measuring cup. Another solution of 2 equivalents of dimethyl phosphite (40 mass\%) was prepared in $\mathrm{MeOH}$ and transferred to a second measuring cup. Both measuring cups were connected to the CPC College System. The flow rate was adjusted to $0.3 \mathrm{~mL} / \mathrm{min}$.pump resulting in a residence time of $78 \mathrm{~min}$. The residence time $\left(\mathrm{t}_{\mathrm{r}}\right)$ was calculated by the following formula: $t_{r}=V_{\text {total }} / r_{\text {total }}$ with $r_{\text {total }}=0.6 \mathrm{~mL} / \mathrm{min}$. At the outlet, the end product was collected at steady state conditions, i.e. after $1.6 * \mathrm{t}_{\mathrm{r}}$. About $10 \mathrm{~mL}$ was collected for analysis. The work up of the reaction mixture was performed by means of the same acid/base extraction as in the batch procedure. The conversion was calculated from the ${ }^{1} \mathrm{H}-\mathrm{NMR}$ spectrum of the reaction mixture before workup.

Compound characterization. Dimethyl (isopropylamino)(phenyl)methylphosphonate (2a). ${ }^{1} \mathrm{H}-\mathrm{NMR}\left(\mathrm{CDCl}_{3}\right) \delta=1.00\left(3 \mathrm{H}, \mathrm{d}, \mathrm{J}=6.1 \mathrm{~Hz}, \mathrm{CH}_{3}(\mathrm{i}-\mathrm{Pr})\right) ; 1.02\left(3 \mathrm{H}, \mathrm{d}, \mathrm{J}=6.3 \mathrm{~Hz}, \mathrm{CH}_{3}(\mathrm{i}-\mathrm{Pr})\right)$; $1.85(1 \mathrm{H}$, br. s, $\mathrm{N} \underline{\mathrm{H}}) ; 2.68(1 \mathrm{H}$, septet, $\mathrm{J}=6.3 \mathrm{~Hz}, \mathrm{C} \underline{\mathrm{H}}(\mathrm{i}-\mathrm{Pr})) ; 3.51\left(3 \mathrm{H}, \mathrm{d}, \mathrm{J}_{\mathrm{H}-\mathrm{P}}=10.5 \mathrm{~Hz}, \mathrm{CH}_{3} \mathrm{O}\right)$; $3.78\left(3 \mathrm{H}, \mathrm{d}, \mathrm{J}_{\mathrm{H}-\mathrm{P}}=10.5 \mathrm{~Hz}, \mathrm{CH}_{3} \mathrm{O}\right) ; 4.17\left(1 \mathrm{H}, \mathrm{d}, \mathrm{J}_{\mathrm{H}-\mathrm{P}}=22.3 \mathrm{~Hz}, \mathrm{C} \underline{\mathrm{H}}-\mathrm{P}\right) ; 7.27-7.43(5 \mathrm{H}, \mathrm{m}$, $\mathrm{C} \underline{\mathrm{H}}(\mathrm{Ph})) ;{ }^{13} \mathrm{C}-\mathrm{NMR}\left(\mathrm{CDCl}_{3}\right) \delta=21.31\left(\underline{\mathrm{CH}}_{3}(\mathrm{i}-\mathrm{Pr})\right) ; 24.04\left(\underline{\mathrm{CH}}_{3}(\mathrm{i}-\mathrm{Pr})\right) ; 45.71\left(\underline{\mathrm{CH}}(\mathrm{i}-\mathrm{Pr}), \mathrm{J}_{\mathrm{C}-\mathrm{P}}\right.$ $=16,2 \mathrm{~Hz}) ; 53,51\left(\underline{\mathrm{CH}}_{3} \mathrm{O}, \mathrm{J}_{\mathrm{C}-\mathrm{P}}=6.9 \mathrm{~Hz}\right) ; 54.00\left(\underline{\mathrm{CH}}_{3} \mathrm{O}, \mathrm{J}_{\mathrm{C}-\mathrm{P}}=6.9 \mathrm{~Hz}\right) ; 57.98\left(\underline{\mathrm{CH}}-\mathrm{P}, \mathrm{J}_{\mathrm{C}-\mathrm{P}}=154.6\right.$ $\mathrm{Hz}) ; 127.99 ; 128.43 ; 128.51 ; 128.61(\underline{\mathrm{CH}}(\mathrm{Ph})) ; 136.29\left(\underline{\mathrm{C}}_{\mathrm{q}}\right) ; \operatorname{IR} 1026$ (P-O), 1066 (P-O), 1241 $(\mathrm{P}=\mathrm{O}), 3303(\mathrm{NH}) ;{ }^{31} \mathrm{P}-\mathrm{NMR}\left(\mathrm{CDCl}_{3}\right) \delta=27.0$; MS $258\left(\mathrm{M}^{+}+1\right)$; HRMS Calc. for $\mathrm{C}_{12} \mathrm{H}_{20} \mathrm{NO}_{3} \mathrm{P}$ $+\mathrm{H}^{+}: 258.1254$, found: $258.1249 ; m p 72.7^{\circ} \mathrm{C}$; white solid.

Diethyl (isopropylamino)(phenyl)methylphosphonate (2b). ${ }^{1} \mathrm{H}-\mathrm{NMR}\left(\mathrm{CDCl}_{3}\right) \delta=0.99(3 \mathrm{H}, \mathrm{d}$, $\left.\mathrm{J}=6.1 \mathrm{~Hz}, \mathrm{CH}_{3}(\mathrm{i}-\mathrm{Pr})\right) ; 1.01\left(3 \mathrm{H}, \mathrm{d}, \mathrm{J}=6.3 \mathrm{~Hz}, \underline{\mathrm{C}}_{3}(\mathrm{i}-\mathrm{Pr})\right) ; 1.11\left(3 \mathrm{H}, \mathrm{t}, \mathrm{J}=7.0 \mathrm{~Hz}, \mathrm{C}_{3} \mathrm{CH}_{2} \mathrm{O}\right)$; $1.30\left(3 \mathrm{H}, \mathrm{t}, \mathrm{J}=7.0 \mathrm{~Hz}, \mathrm{C}_{3} \mathrm{CH}_{2} \mathrm{O}\right) ; 1.80(1 \mathrm{H}$, br. s, $\mathrm{N} \underline{\mathrm{H}}) ; 2.68(1 \mathrm{H}$, septet, J = 6.2 Hz, $\mathrm{CH}(\mathrm{i}-\mathrm{Pr})$ ); 3.70-3.83 (1H, m, $\left.\mathrm{CH}_{3} \mathrm{CH}_{2} \mathrm{O}\right)$; 3.88-4.04 (1H, m $\left.\mathrm{CH}_{3} \underline{\mathrm{C}}_{2} \mathrm{O}\right)$; 4.06-4.22 (3H, m, $\mathrm{CH}_{3} \mathrm{C}_{2} \mathrm{O}, \mathrm{C} \underline{\mathrm{H}}-$ $\mathrm{P}) ; 7.25-7.42(5 \mathrm{H}, \mathrm{m}, \mathrm{C} \underline{\mathrm{H}}(\mathrm{Ph})) ;{ }^{13} \mathrm{C}-\mathrm{NMR}\left(\mathrm{CDCl}_{3}\right) \delta=16.24\left(\mathrm{CH}_{3} \mathrm{CH}_{2} \mathrm{O}, \mathrm{J}_{\mathrm{C}-\mathrm{P}}=5.8 \mathrm{~Hz}\right) ; 16.47$ 


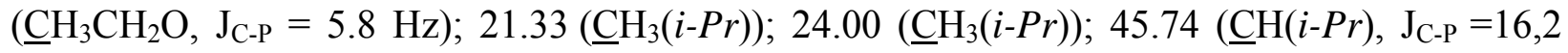
$\mathrm{Hz}) ; 58.30\left(\underline{\mathrm{CH}}-\mathrm{P}, \mathrm{J}_{\mathrm{C}-\mathrm{P}}=153.5 \mathrm{~Hz}\right) ; 62.68\left(\mathrm{CH}_{3} \mathrm{CH}_{2} \mathrm{O}, \mathrm{J}_{\mathrm{C}-\mathrm{P}}=6.9 \mathrm{~Hz}\right) ; 63.12\left(\mathrm{CH}_{3} \underline{\mathrm{CH}}_{2} \mathrm{O}, \mathrm{J}_{\mathrm{C}-\mathrm{P}}=\right.$ $6.9 \mathrm{~Hz}) ; 127.72 ; 128.42 ; 128.50(\underline{\mathrm{CH}}(\mathrm{Ph})) ; 136.63\left(\underline{\mathrm{C}}_{\mathrm{q}}\right) ; \operatorname{IR} 1028(\mathrm{P}-\mathrm{O}), 1061(\mathrm{P}-\mathrm{O}), 1240(\mathrm{P}=\mathrm{O})$, $3294(\mathrm{NH}) ;{ }^{31} \mathrm{P}-\mathrm{NMR}\left(\mathrm{CDCl}_{3}\right) \delta=24.74$; MS $286\left(\mathrm{M}^{+}+1\right)$; HRMS Calc. for $\mathrm{C}_{14} \mathrm{H}_{24} \mathrm{NO}_{3} \mathrm{P}+\mathrm{H}^{+}$: 286.1566, found: $286.1561 ; m p 36.3{ }^{\circ} \mathrm{C}$; white solid.

Diisopropyl (isopropylamino)(phenyl)methylphosphonate (2c). ${ }^{1} \mathrm{H}-\mathrm{NMR}\left(\mathrm{CDCl}_{3}\right) \delta=0.94$ $\left(3 \mathrm{H}, \mathrm{d}, \mathrm{J}=6.3 \mathrm{~Hz}, \underline{\mathrm{H}}_{3} \mathrm{CHO}\right) ; 0.98\left(3 \mathrm{H}, \mathrm{d}, \mathrm{J}=6.1 \mathrm{~Hz}, \mathrm{C}_{3}(i-P r)\right) ; 1.00(3 \mathrm{H}, \mathrm{d}, \mathrm{J}=6.1 \mathrm{~Hz}$, $\left.\mathrm{C}_{3}(i-P r)\right) ; 1.22\left(3 \mathrm{H}, \mathrm{d}, \mathrm{J}=6.3 \mathrm{~Hz}, \underline{\mathrm{CH}}_{3} \mathrm{CHO}\right) ; 1.30\left(6 \mathrm{H}, \mathrm{d}, \mathrm{J}=6.1 \mathrm{~Hz}, \underline{\mathrm{C}}_{3} \mathrm{CHO}\right) ; 1.79$ (1H, br. $\mathrm{s}, \mathrm{N} \underline{\mathrm{H}}) ; 2.67(1 \mathrm{H}$, septet, J = 6.2 Hz, C$(i-P r)) ; 4.05\left(1 \mathrm{H}, \mathrm{d}, \mathrm{J}_{\mathrm{H}-\mathrm{P}}=22.3 \mathrm{~Hz}, \mathrm{C} \underline{\mathrm{H}}-\mathrm{P}\right) ; 4.47(1 \mathrm{H}, \mathrm{d} x$ septet, $\left.\mathrm{J}_{\mathrm{H}-\mathrm{P}}=7.2 \mathrm{~Hz}, \mathrm{~J}_{2}=6.2 \mathrm{~Hz}, \mathrm{CH}_{3} \mathrm{CHO}\right) ; 4.73\left(1 \mathrm{H}, \mathrm{d}\right.$ x septet, $\mathrm{J}_{\mathrm{H}-\mathrm{P}}=7.4 \mathrm{~Hz}, \mathrm{~J}_{2}=6.2 \mathrm{~Hz}$, $\left.\mathrm{CH}_{3} \mathrm{CHO}\right) ; 7.23-7.42(5 \mathrm{H}, \mathrm{m}, \mathrm{C} \underline{\mathrm{H}}(\mathrm{Ph})) ;{ }^{13} \mathrm{C}-\mathrm{NMR}\left(\mathrm{CDCl}_{3}\right) \delta=21.38\left(\underline{\mathrm{CH}}_{3}(\mathrm{i}-\mathrm{Pr})\right) ; 23.33$ $\left(\underline{\mathrm{CH}}_{3} \mathrm{CHO}, \mathrm{J}_{\mathrm{C}-\mathrm{P}}=5.8 \mathrm{~Hz}\right) ; 23.87\left(\underline{\mathrm{CH}}_{3} \mathrm{CHO}, \mathrm{J}_{\mathrm{C}-\mathrm{P}}=5.8 \mathrm{~Hz}\right) ; 24.09\left(\underline{\mathrm{CH}}_{3}(i-P r)\right) ; 24.25\left(\underline{\mathrm{CH}}_{3} \mathrm{CHO}\right.$, $\left.\mathrm{J}_{\mathrm{C}-\mathrm{P}}=3.5 \mathrm{~Hz}\right) ; 24.30\left(\underline{\mathrm{CH}}_{3} \mathrm{CHO}, \mathrm{J}_{\mathrm{C}-\mathrm{P}}=3.5 \mathrm{~Hz}\right) ; 45.78\left(\underline{\mathrm{CH}}(\mathrm{i}-\mathrm{Pr}), \mathrm{J}_{\mathrm{C}-\mathrm{P}}=16.2 \mathrm{~Hz}\right) ; 58.72(\underline{\mathrm{C}} \mathrm{H}-\mathrm{P}$, $\left.\mathrm{J}_{\mathrm{C}-\mathrm{P}}=155.8 \mathrm{~Hz}\right) ; 71.10\left(\mathrm{CH}_{3} \underline{\mathrm{CHO}}, \mathrm{J}_{\mathrm{C}-\mathrm{P}}=6.9 \mathrm{~Hz}\right) ; 71.42\left(\mathrm{CH}_{3} \underline{\mathrm{CHO}}, \mathrm{J}_{\mathrm{C}-\mathrm{P}}=6.9 \mathrm{~Hz}\right) ; 127.50$; 127.54; 128.25; $128.60 ; 128.67(\underline{\mathrm{CH}}(\mathrm{Ph})) ; 137.13\left(\underline{\mathrm{C}}_{\mathrm{q}}, \mathrm{J}_{\mathrm{C}-\mathrm{P}}=2.3 \mathrm{~Hz}\right) ; \mathrm{IR} 985(\mathrm{P}-\mathrm{O}), 1010(\mathrm{P}-\mathrm{O})$, $1241(\mathrm{P}=\mathrm{O}), 3294(\mathrm{NH}) ;{ }^{31} \mathrm{P}-\mathrm{NMR}\left(\mathrm{CDCl}_{3}\right) \delta=23.18 ;$ MS $314\left(\mathrm{M}^{+}+1\right)$; HRMS Calc. for $\mathrm{C}_{16} \mathrm{H}_{28} \mathrm{NO}_{3} \mathrm{P}+\mathrm{H}^{+}: 314.1880$, found: $314.1883 ; m p 20-25\left({ }^{\circ} \mathrm{C}\right)$, white solid.

Dimethyl (tert-butylamino)(phenyl)methylphosphonate (2d) $.{ }^{1} \mathrm{H}-\mathrm{NMR}\left(\mathrm{CDCl}_{3}\right) \delta=0.99(9 \mathrm{H}$,

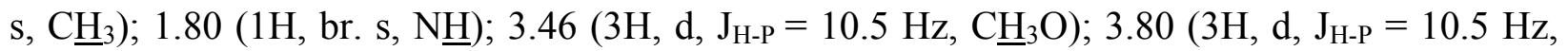
$\left.\mathrm{C}_{3} \mathrm{O}\right) ; 4.18\left(1 \mathrm{H}, \mathrm{d}, \mathrm{J}_{\mathrm{H}-\mathrm{P}}=25,0 \mathrm{~Hz}, \mathrm{CH}-\mathrm{P}\right) ; 7.23-7.46(5 \mathrm{H}, \mathrm{m}, \mathrm{CH}(\mathrm{Ph})) ;{ }^{13} C-N M R\left(C D C l_{3}\right) \delta=$ $29.96\left(\underline{\mathrm{CH}}_{3}\right) ; 52.36\left(\underline{\mathrm{C}}_{\mathrm{q}}, \mathrm{J}_{\mathrm{C}-\mathrm{P}}=15.0 \mathrm{~Hz}\right) ; 53.20\left(\underline{\mathrm{CH}}_{3} \mathrm{O}, \mathrm{J}_{\mathrm{C}-\mathrm{P}}=8.1 \mathrm{~Hz}\right) ; 54.49\left(\underline{\mathrm{CH}}_{3} \mathrm{O}, \mathrm{J}_{\mathrm{C}-\mathrm{P}}=5.8\right.$ $\mathrm{Hz}) ; 55.48\left(\underline{\mathrm{CH}}-\mathrm{P}, \mathrm{J}_{\mathrm{C}-\mathrm{P}}=155.8 \mathrm{~Hz}\right) ; 127.46 ; 127.51 ; 128.16 ; 128.24 ; 128.35 ; 128.38(\underline{\mathrm{CH}}(\mathrm{Ph}))$; $139.54\left(\underline{\mathrm{C}}_{\mathrm{q}}\right) ; \operatorname{IR} 1031(\mathrm{P}-\mathrm{O}), 1065(\mathrm{P}-\mathrm{O}), 1240(\mathrm{P}=\mathrm{O}), 3310(\mathrm{NH}) ;{ }^{31} \mathrm{P}-\mathrm{NMR}\left(\mathrm{CDCl}_{3}\right) \delta=27.05$; MS $272\left(\mathrm{M}^{+}+1\right)$; HRMS Calc. for $\mathrm{C}_{13} \mathrm{H}_{22} \mathrm{NO}_{3} \mathrm{P}+\mathrm{H}^{+}$: 272.1410, found: 272.1405 ; $\mathrm{mp} 92.5^{\circ} \mathrm{C}$; white solid.

Dimethyl (benzylamino)(phenyl)methylphosphonate (2e). ${ }^{1} \mathrm{H}-N M R\left(C D C l_{3}\right) \delta=2.41(1 \mathrm{H}$, br. $\mathrm{s}, \mathrm{N} \underline{\mathrm{H}}) ; 3.54\left(3 \mathrm{H}, \mathrm{d}, \mathrm{J}_{\mathrm{H}-\mathrm{P}}=10.5 \mathrm{~Hz}, \underline{\mathrm{CH}}_{3} \mathrm{O}\right) ; 3.74\left(3 \mathrm{H}, \mathrm{d}, \mathrm{J}_{\mathrm{H}-\mathrm{P}}=10.5 \mathrm{~Hz}, \mathrm{CH}_{3} \mathrm{O}\right) ; 3.55\left(1 \mathrm{H}, \mathrm{d}, \mathrm{J}_{\mathrm{AB}}\right.$ $\left.=13.2 \mathrm{~Hz}, \underline{\mathrm{C}}_{2} \mathrm{~N}\right) ; 3.82\left(1 \mathrm{H}, \mathrm{d}, \mathrm{J}_{\mathrm{AB}}=13.2 \mathrm{~Hz}, \underline{\mathrm{C}}_{2} \mathrm{~N}\right) ; 4.05\left(1 \mathrm{H}, \mathrm{d}, \mathrm{J}_{\mathrm{H}-\mathrm{P}}=20.1 \mathrm{~Hz}, \mathrm{C} \underline{\mathrm{H}}-\mathrm{P}\right) ; 7.22-$ $7.45(5 \mathrm{H}, \mathrm{m}, \mathrm{CH}(\mathrm{Ph})) ;{ }^{13} \mathrm{C}-\mathrm{NMR}\left(\mathrm{CDCl}_{3}\right) \delta=51.14\left(\underline{\mathrm{CH}}_{2} \mathrm{~N}, \mathrm{~J}_{\mathrm{C}-\mathrm{P}}=17.31 \mathrm{~Hz}\right) ; 53.40\left(\underline{\mathrm{CH}}_{3} \mathrm{O}, \mathrm{J}_{\mathrm{C}-\mathrm{P}}\right.$ $=5.8 \mathrm{~Hz}) ; 53.71\left(\underline{\mathrm{CH}}_{3} \mathrm{O}, \mathrm{J}_{\mathrm{C}-\mathrm{P}}=6.9 \mathrm{~Hz}\right) ; 59.29\left(\underline{\mathrm{CH}}-\mathrm{P}, \mathrm{J}_{\mathrm{C}-\mathrm{P}}=154.6 \mathrm{~Hz}\right) ; 128.02 ; 128.07 ; 128.33$; $128.39 ; 128.56 ; 128.59 ; 128.63(\mathrm{CH}(\mathrm{Ph})) ; 135.50\left(\mathrm{C}_{\mathrm{q}}, \mathrm{J}_{\mathrm{C}-\mathrm{P}}=3.5 \mathrm{~Hz}\right) ; 139.18\left(\underline{\mathrm{C}}_{\mathrm{q}} \mathrm{CH}_{2}\right) ; \operatorname{IR} 1034$ $(\mathrm{P}-\mathrm{O}), 1230(\mathrm{P}=\mathrm{O}), 3437(\mathrm{NH}) ;{ }^{31} \mathrm{P}-\mathrm{NMR}\left(\mathrm{CDCl}_{3}\right) \delta=26.44 ; \mathrm{MS} 306\left(\mathrm{M}^{+}+1\right)$; HRMS Calc. for $\mathrm{C}_{16} \mathrm{H}_{20} \mathrm{NO}_{3} \mathrm{P}+\mathrm{H}^{+}: 306.1254$, found 306.1249; colourless oil.

Dimethyl (4-chlorophenyl)(isopropylamino)methylphosphonate(2f). ${ }^{1} H-N M R\left(C D C l_{3}\right) \delta=$ $0.99\left(3 \mathrm{H}, \mathrm{d}, \mathrm{J}=6.2 \mathrm{~Hz}, \mathrm{CH}_{3}(\mathrm{i}-\mathrm{Pr})\right) ; 1.01$ (3H, d, J = 6.2 Hz, $\left.\underline{\mathrm{H}}_{3}(i-P r)\right) ; 1.76$ (1H, br. s, N $\left.\underline{\mathrm{H}}\right)$; $2.65(1 \mathrm{H}$, septet, $\mathrm{J}=6.2 \mathrm{~Hz}, \mathrm{CH}(\mathrm{i}-\mathrm{Pr})) ; 3.57\left(3 \mathrm{H}, \mathrm{d}, \mathrm{J}_{\mathrm{H}-\mathrm{P}}=10.5 \mathrm{~Hz}, \mathrm{CH}_{3} \mathrm{O}\right) ; 3.77\left(3 \mathrm{H}, \mathrm{d}, \mathrm{J}_{\mathrm{H}-\mathrm{P}}=\right.$ $\left.10.5 \mathrm{~Hz}, \mathrm{CH}_{3} \mathrm{O}\right) ; 4.15\left(1 \mathrm{H}, \mathrm{d}, \mathrm{J}_{\mathrm{H}-\mathrm{P}}=22.0 \mathrm{~Hz}, \mathrm{C} \underline{\mathrm{H}}-\mathrm{P}\right) ; 7.31-7.38(4 \mathrm{H}, \mathrm{m}, \mathrm{C} \underline{\mathrm{H}}(\mathrm{Ph})) ;{ }^{13} \mathrm{C}-\mathrm{NMR}$ $\left(C D C l_{3}\right) \delta=21.25\left(\underline{C H}_{3}(i-P r)\right) ; 23.93\left(\underline{C H}_{3}(i-P r)\right) ; 45.85\left(\underline{\mathrm{CH}}(i-P r), J_{\mathrm{C}-\mathrm{P}}=16.15 \mathrm{~Hz}\right) ; 53.47$ $\left(\underline{\mathrm{CH}}_{3} \mathrm{O}, \mathrm{J}_{\mathrm{C}-\mathrm{P}}=6.9 \mathrm{~Hz}\right) ; 54.02\left(\underline{\mathrm{CH}}_{3} \mathrm{O}, \mathrm{J}_{\mathrm{C}-\mathrm{P}}=6.9 \mathrm{~Hz}\right) ; 57.35\left(\underline{\mathrm{CH}}-\mathrm{P}, \mathrm{J}_{\mathrm{C}-\mathrm{P}}=154.6 \mathrm{~Hz}\right) ; 128.71$; $128.74 ; 129.70 ; 129.78(\underline{\mathrm{CH}}(\mathrm{Ph})) ; 133.66\left(\underline{\mathrm{C}}_{\mathrm{q}}, \mathrm{J}_{\mathrm{C}-\mathrm{P}}=3.5 \mathrm{~Hz}\right) ; 135.00\left(\underline{\mathrm{C}}_{\mathrm{q}}, \mathrm{J}_{\mathrm{C}-\mathrm{P}}=3.5 \mathrm{~Hz}\right) ; I R 1030$ 
(P-O), $1062(\mathrm{P}-\mathrm{O}), 1244(\mathrm{P}=\mathrm{O}), 3292(\mathrm{NH}) ;{ }^{31} \mathrm{P}-\mathrm{NMR}\left(\mathrm{CDCl}_{3}\right) \delta=26.37 ; M S 292\left(\mathrm{M}^{+}+1\right)$; HRMS Calc. for $\mathrm{C}_{12} \mathrm{H}_{19} \mathrm{ClNO}_{3} \mathrm{P}+\mathrm{H}^{+}$: 292.0864, found: $292.0871 ; m p 56.1^{\circ} \mathrm{C}$; yellow solid.

Dimethyl (isopropylamino)(4-methylphenyl)methylphosphonate (2g). ${ }^{1} \mathrm{H}-\mathrm{NMR}\left(\mathrm{CDCl}_{3}\right) \delta=$

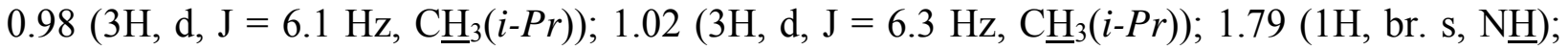
$2.34\left(3 \mathrm{H}, \mathrm{d}, \mathrm{J}=1.7 \mathrm{~Hz}, \underline{\mathrm{C}}_{3}(\mathrm{Ph})\right) ; 2.67(1 \mathrm{H}$, septet, J = 6.1 Hz, $\underline{\mathrm{CH}}(\mathrm{i}-\mathrm{Pr})) ; 3.52\left(3 \mathrm{H}, \mathrm{d}, \mathrm{J}_{\mathrm{H}-\mathrm{P}}=\right.$ $\left.10.5 \mathrm{~Hz}, \mathrm{C}_{3} \mathrm{O}\right) ; 3.77\left(3 \mathrm{H}, \mathrm{d}, \mathrm{J}_{\mathrm{H}-\mathrm{P}}=10.5 \mathrm{~Hz}, \mathrm{CH}_{3} \mathrm{O}\right) ; 4.14\left(1 \mathrm{H}, \mathrm{d}, \mathrm{J}_{\mathrm{H}-\mathrm{P}}=21.7 \mathrm{~Hz}, \mathrm{C} \underline{\mathrm{H}}-\mathrm{P}\right) ; 7.17$ $(2 \mathrm{H}, \mathrm{d}, \mathrm{J}=8.3 \mathrm{~Hz}, \mathrm{C} \underline{\mathrm{H}}(\mathrm{Ph})) ; 7.27-7.30(2 \mathrm{H}, \sim \mathrm{dd}, \mathrm{C} \underline{\mathrm{H}}(\mathrm{Ph})) ;{ }^{13} \mathrm{C}-\mathrm{NMR}\left(\mathrm{CDCl}_{3}\right) \delta=21.15\left(\underline{\mathrm{CH}}_{3}(\mathrm{i}-\right.$ Pr), $\left.\underline{\mathrm{CH}}_{3}(\mathrm{Ph})\right) ; 21.21\left(\underline{\mathrm{CH}}_{3}(i-P r)\right) ; 45.52\left(\underline{\mathrm{C}}(\mathrm{i}-\mathrm{Pr}), \mathrm{J}_{\mathrm{C}-\mathrm{P}}=16,2 \mathrm{~Hz}\right) ; 53,39\left(\underline{\mathrm{CH}}_{3} \mathrm{O}, \mathrm{J}_{\mathrm{C}-\mathrm{P}}=8.1 \mathrm{~Hz}\right)$; $53.85\left(\underline{\mathrm{CH}}_{3} \mathrm{O}, \mathrm{J}_{\mathrm{C}-\mathrm{P}}=6.9 \mathrm{~Hz}\right) ; 57.57\left(\underline{\mathrm{CH}}-\mathrm{P}, \mathrm{J}_{\mathrm{C}-\mathrm{P}}=155,8 \mathrm{~Hz}\right) ; 128.21 ; 128.30 ; 129.25 ; 129.28$ $(\underline{\mathrm{C}} \mathrm{H}(\mathrm{Ph})) ; 133.12\left(\underline{\mathrm{C}}_{\mathrm{q}}-\mathrm{CH}_{3}\right), 137.56\left(\underline{\mathrm{C}}_{\mathrm{q}}-\mathrm{CH}\right) ; \mathrm{IR} 1033(\mathrm{P}-\mathrm{O}), 1067(\mathrm{P}-\mathrm{O}), 1245(\mathrm{P}=\mathrm{O}), 3299$ $(\mathrm{NH}) ;{ }^{31} \mathrm{P}-\mathrm{NMR}\left(\mathrm{CDCl}_{3}\right) \delta=27.20 ;$ MS $272\left(\mathrm{M}^{+}+1\right)$; HRMS Calc. for $\mathrm{C}_{13} \mathrm{H}_{22} \mathrm{NO}_{3} \mathrm{P}+\mathrm{H}^{+}$: 272.1410, found $272.1417 ; \mathrm{mp} 73.5\left({ }^{\circ} \mathrm{C}\right)$; white solid.

Dimethyl (isopropylamino)(4-nitrophenyl)methylphosphonate (2h). ${ }^{1} \mathrm{H}-\mathrm{NMR}\left(\mathrm{CDCl}_{3}\right) \delta=$

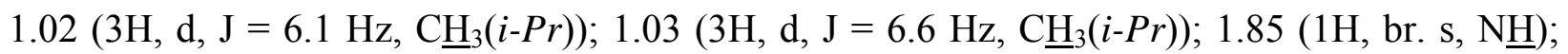
$2.64(1 \mathrm{H}$, septet, $\mathrm{J}=6.2 \mathrm{~Hz}, \mathrm{C} \underline{\mathrm{H}}(\mathrm{i}-\mathrm{Pr})) ; 3.64\left(3 \mathrm{H}, \mathrm{d}, \mathrm{J}_{\mathrm{H}-\mathrm{P}}=10.7 \mathrm{~Hz}, \mathrm{CH}_{3} \mathrm{O}\right) ; 3.79\left(3 \mathrm{H}, \mathrm{d}, \mathrm{J}_{\mathrm{H}-\mathrm{P}}=\right.$ $\left.10.7 \mathrm{~Hz}, \mathrm{CH}_{3} \mathrm{O}\right) ; 4.32\left(1 \mathrm{H}, \mathrm{d}, \mathrm{J}_{\mathrm{H}-\mathrm{P}}=22.6 \mathrm{~Hz}, \mathrm{C} \underline{\mathrm{H}}-\mathrm{P}\right) ; 7.62\left(2 \mathrm{H}, \mathrm{dd}, \mathrm{J}=8.8 \mathrm{~Hz}, \mathrm{~J}_{\mathrm{H}-\mathrm{P}}=2.2 \mathrm{~Hz}\right.$, $\mathrm{C} \underline{\mathrm{H}}(\mathrm{Ph})) ; 8.21(2 \mathrm{H}, \mathrm{d}, \mathrm{J}=8.5 \mathrm{~Hz}, \mathrm{C} \underline{\mathrm{H}}(\mathrm{Ph})) ;{ }^{13} \mathrm{C}-N M R\left(\mathrm{CDCl}_{3}\right) \delta=21.33\left(\underline{\mathrm{CH}}_{3}(\mathrm{i}-\mathrm{Pr})\right) ; 23.94$ $\left(\underline{\mathrm{CH}}_{3}(\mathrm{i}-\mathrm{Pr})\right) ; 45.47\left(\underline{\mathrm{C}} \mathrm{H}(\mathrm{i}-\mathrm{Pr}), \mathrm{J}_{\mathrm{C}-\mathrm{P}}=15.00 \mathrm{~Hz}\right) ; 53.58\left(\underline{\mathrm{CH}}_{3} \mathrm{O}, \mathrm{J}_{\mathrm{C}-\mathrm{P}}=6.9 \mathrm{~Hz}\right) ; 54.14\left(\underline{\mathrm{CH}}_{3} \mathrm{O}, \mathrm{J}_{\mathrm{C}-\mathrm{P}}=\right.$ $6.9 \mathrm{~Hz}) ; 57.82\left(\underline{\mathrm{CH}}-\mathrm{P}, \mathrm{J}_{\mathrm{C}-\mathrm{P}}=152.3 \mathrm{~Hz}\right) ; 123.64 ; 123.67 ; 129.18 ; 129.26(\underline{\mathrm{CH}}(\mathrm{Ph})) ; 144.53\left(\underline{\mathrm{C}}_{\mathrm{q}}\right.$, $\left.\mathrm{J}_{\mathrm{C}-\mathrm{P}}=3.5 \mathrm{~Hz}\right) ; 147.60\left(\mathrm{C}_{\mathrm{q}}-\mathrm{NO}_{2}\right)$; IR $1038(\mathrm{P}-\mathrm{O}), 1057(\mathrm{P}-\mathrm{O}), 1257(\mathrm{P}=\mathrm{O}), 1346,3297(\mathrm{NH}) ;{ }^{31} \mathrm{P}-$ NMR $\left(C D C l_{3}\right) \delta=25.17$; MS $301\left(\mathrm{M}^{-}-1\right)$; HRMS Calc. for $\mathrm{C}_{12} \mathrm{H}_{19} \mathrm{~N}_{2} \mathrm{O}_{5} \mathrm{P}-\mathrm{H}^{+}: 301.0959$, found 301.0956; $m p 102.6{ }^{\circ} \mathrm{C}$; orange solid.

Dimethyl (allylamino)(2-furyl)methylphosphonate (2i). ${ }^{1} \mathrm{H}-\mathrm{NMR}\left(\mathrm{CDCl}_{3}\right) \delta=2.04(1 \mathrm{H}$, br. s, $\mathrm{N} \underline{\mathrm{H}}) ; 2.95\left(1 \mathrm{H}, \mathrm{dd}, \mathrm{J}_{\mathrm{AB}}=13.8 \mathrm{~Hz}, \mathrm{~J}_{2}=6.7 \mathrm{~Hz}, \underline{\mathrm{H}}_{2}\right) ; 3.17\left(1 \mathrm{H}, \mathrm{d}, \mathrm{J}_{\mathrm{AB}}=13.8 \mathrm{~Hz}, \mathrm{~J}_{2}=5.4 \mathrm{~Hz}, \mathrm{~J}_{3}\right.$ $\left.=1.4 \mathrm{~Hz}, \mathrm{C}_{2}\right) ; 3.53\left(3 \mathrm{H}, \mathrm{d}, \mathrm{J}_{\mathrm{H}-\mathrm{P}}=10.7 \mathrm{~Hz}, \mathrm{C}_{3} \mathrm{O}\right) ; 3.69\left(3 \mathrm{H}, \mathrm{d}, \mathrm{J}_{\mathrm{H}-\mathrm{P}}=10.7 \mathrm{~Hz}, \mathrm{C}_{3} \mathrm{O}\right) ; 4.06$ $\left(1 \mathrm{H}, \mathrm{d}, \mathrm{J}_{\mathrm{H}-\mathrm{P}}=22.3 \mathrm{~Hz}, \mathrm{C} \underline{\mathrm{H}}-\mathrm{P}\right) ; 4.96-5.18\left(2 \mathrm{H}, \mathrm{m},=\mathrm{CH}_{2}\right) ; 5.68\left(1 \mathrm{H}, \mathrm{J}_{1}=17.1 \mathrm{~Hz}, \mathrm{~J}_{2}=10.2 \mathrm{~Hz}, \mathrm{~J}_{3}\right.$ $\left.=6.8 \mathrm{~Hz}, \mathrm{~J}_{4}=5.5 \mathrm{~Hz}, \mathrm{CH}_{2} \mathrm{CH}=\right) ; 6.23-6.27(2 \mathrm{H}, \mathrm{m},=\mathrm{C} \underline{\mathrm{H}}) ; 7.30-7.32(1 \mathrm{H}, \mathrm{m}, \mathrm{CHO}) ;{ }^{13} C-N M R$ $\left(C D C l_{3}\right) \delta=50.13\left(\underline{\mathrm{CH}}_{2}, \mathrm{~J}_{\mathrm{C}-\mathrm{P}}=16.2 \mathrm{~Hz}\right) ; 52.80\left(\underline{\mathrm{CH}}-\mathrm{P}, \mathrm{J}_{\mathrm{C}-\mathrm{P}}=162.7 \mathrm{~Hz}\right) ; 53.46\left(\underline{\mathrm{CH}}_{3} \mathrm{O}, \mathrm{J}_{\mathrm{C}-\mathrm{P}}=5.8\right.$ $\mathrm{Hz}) ; 53.84\left(\underline{\mathrm{CH}}_{3} \mathrm{O}, \mathrm{J}_{\mathrm{C}-\mathrm{P}}=6.9 \mathrm{~Hz}\right) ; 109.43\left(=\underline{\mathrm{CHC}} \mathrm{q}_{\mathrm{q}} \mathrm{O}, \mathrm{J}_{\mathrm{C}-\mathrm{P}}=8.1 \mathrm{~Hz}\right) ; 110.65(\underline{\mathrm{CH}}=\mathrm{CHO}) ; 117.20$ $\left(=\underline{\mathrm{CH}}_{2}\right) ; 135.58\left(\mathrm{CH}_{2} \underline{\mathrm{CH}}=\right) ; 142.71\left(=\underline{\mathrm{CHO}}, \mathrm{J}_{\mathrm{C}-\mathrm{P}}=2.3 \mathrm{~Hz}\right) ; 149.37\left(=\underline{\mathrm{C}}_{\mathrm{q}} \mathrm{O}, \mathrm{J}_{\mathrm{C}-\mathrm{P}}=2.3 \mathrm{~Hz}\right) ; I R$ $1044(\mathrm{P}-\mathrm{O}) ; 1248(\mathrm{P}=\mathrm{O}) ; 1643(\mathrm{C}=\mathrm{C}) ; 3317(\mathrm{NH}) ;{ }^{31} \mathrm{P}-\mathrm{NMR}\left(\mathrm{CDCl}_{3}\right) \delta=24.16 ; M S 246$ $\left(\mathrm{M}^{+}+1\right) ; 136\left(\mathrm{M}^{+}-\mathrm{P}(\mathrm{O})(\mathrm{OMe})_{2}+1\right)$; HRMS Calc. for $\mathrm{C}_{10} \mathrm{H}_{16} \mathrm{NO}_{4} \mathrm{P}+\mathrm{H}^{+}: 246.0890$, found 246.0884; brown oil.

Dimethyl (benzylamino)(2-furyl)methylphosphonate (2j). ${ }^{1} \mathrm{H}$-NMR $\left(\mathrm{CDCl}_{3}\right) \delta=2.17(1 \mathrm{H}$, br. $\mathrm{s}, \mathrm{N} \underline{\mathrm{H}}) ; 3.60\left(1 \mathrm{H}, \mathrm{d}, \mathrm{J}_{\mathrm{AB}}=13.2 \mathrm{~Hz}, \underline{\mathrm{C}}_{2}\right) ; 3.63\left(3 \mathrm{H}, \mathrm{d}, \mathrm{J}_{\mathrm{H}-\mathrm{P}}=10.7 \mathrm{~Hz}, \underline{\mathrm{C}}_{3} \mathrm{O}\right) ; 3.81\left(3 \mathrm{H}, \mathrm{d}, \mathrm{J}_{\mathrm{H}-\mathrm{P}}=\right.$ $\left.10.5 \mathrm{~Hz}, \underline{\mathrm{C}}_{3} \mathrm{O}\right) ; 3.87\left(1 \mathrm{H}, \mathrm{d}, \mathrm{J}_{\mathrm{AB}}=13.2 \mathrm{~Hz}, \mathrm{C}_{2}\right) ; 4.12\left(1 \mathrm{H}, \mathrm{d}, \mathrm{J}_{\mathrm{H}-\mathrm{P}}=22.3 \mathrm{~Hz}, \mathrm{C} \underline{\mathrm{H}}-\mathrm{P}\right) ; 6.37(2 \mathrm{H}$, $\mathrm{m},=\mathrm{C} \underline{\mathrm{H}}) ; 7.22-7.35(5 \mathrm{H}, \mathrm{m}, \mathrm{C} \underline{\mathrm{H}}(\mathrm{Ph})), 7.45-7.47(1 \mathrm{H}, \mathrm{m}, \mathrm{C} \underline{\mathrm{HO}}) ;{ }^{13} \mathrm{C}-\mathrm{NMR}\left(\mathrm{CDCl}_{3}\right) \delta=$ $51.32\left(\underline{\mathrm{CH}}_{2}, \mathrm{~J}_{\mathrm{C}-\mathrm{P}}=16.2 \mathrm{~Hz}\right) ; 52.71\left(\underline{\mathrm{CH}}-\mathrm{P}, \mathrm{J}_{\mathrm{C}-\mathrm{P}}=161.5 \mathrm{~Hz}\right) ; 53.42\left(\underline{\mathrm{CH}}_{3} \mathrm{O}, \mathrm{J}_{\mathrm{C}-\mathrm{P}}=6.9 \mathrm{~Hz}\right) ; 53.94$ $\left(\underline{\mathrm{CH}}_{3} \mathrm{O}, \mathrm{J}_{\mathrm{C}-\mathrm{P}}=6.9 \mathrm{~Hz}\right) ; 109.57\left(=\underline{\mathrm{CHC}} \mathrm{q}_{\mathrm{q}} \mathrm{O}, \mathrm{J}_{\mathrm{C}-\mathrm{P}}=8.1 \mathrm{~Hz}\right) ; 110.66(\underline{\mathrm{CH}}=\mathrm{CHO}) ; 127.26 ; 128.42$ $(\underline{\mathrm{C}} \mathrm{H}(\mathrm{Ph})) ; 138.86\left(\underline{\mathrm{C}}_{\mathrm{q}}(\mathrm{Ph})\right) ; 142.80(=\underline{\mathrm{C} H O}) ; 149.37\left(=\underline{\mathrm{C}}_{\mathrm{q}} \mathrm{O}\right) ; \mathrm{IR} 1037(\mathrm{P}-\mathrm{O}) ; 1251(\mathrm{P}=\mathrm{O}) ; 3311$; 
$3470(\mathrm{NH}) ;{ }^{31} \mathrm{P}-\mathrm{NMR}\left(\mathrm{CDCl}_{3}\right) \delta=34.01 ;$ MS $296\left(\mathrm{M}^{+}+1\right)$; HRMS Calc. for $\mathrm{C}_{14} \mathrm{H}_{18} \mathrm{NO}_{4} \mathrm{P}+\mathrm{H}^{+}$: 296.1046, found 296.1049; yellow oil.

Dimethyl 1H-indol-3-yl(isopropylamino)methylphosphonate (2k). ${ }^{1} \mathrm{H}-\mathrm{NMR}\left(\mathrm{CDCl}_{3}\right) \delta=0.99$ $\left(3 \mathrm{H}, \mathrm{d}, \mathrm{J}=6.3 \mathrm{~Hz}, \mathrm{CH}_{3}(\mathrm{i}-\mathrm{Pr})\right) ; 1.02\left(3 \mathrm{H}, \mathrm{d}, \mathrm{J}=6.1 \mathrm{~Hz}, \mathrm{C}_{3}(\mathrm{i}-\mathrm{Pr})\right) ; 1.81$ (1H, br. s, N $\left.\underline{\mathrm{H}}\right) ; 2.82$ $(1 \mathrm{H}$, septet, J = 6.2 Hz, $\mathrm{C} \underline{\mathrm{H}}(\mathrm{i}-\mathrm{Pr})) ; 3.48\left(3 \mathrm{H}, \mathrm{d}, \mathrm{J}_{\mathrm{H}-\mathrm{P}}=10.5 \mathrm{~Hz}, \mathrm{CH}_{3} \mathrm{O}\right) ; 3.82\left(3 \mathrm{H}, \mathrm{d}, \mathrm{J}_{\mathrm{H}-\mathrm{P}}=10.5\right.$ $\left.\mathrm{Hz}, \underline{\mathrm{C}}_{3} \mathrm{O}\right) ; 4.51\left(1 \mathrm{H}, \mathrm{d}, \mathrm{J}_{\mathrm{H}-\mathrm{P}}=20.6 \mathrm{~Hz}, \mathrm{C} \underline{\mathrm{H}}-\mathrm{P}\right) ; 7.10-7.26(3 \mathrm{H}, \mathrm{C} \underline{\mathrm{H}}-\mathrm{N}, 2 \mathrm{xC} \underline{\mathrm{H}}(\mathrm{Ph})) ; 7.38(1 \mathrm{H}, \mathrm{d}$, $\mathrm{J}=7.4 \mathrm{~Hz}, \mathrm{C} \underline{\mathrm{H}}(\mathrm{Ph})) ; 7.73(1 \mathrm{H}, \mathrm{d}, \mathrm{J}=7.7 \mathrm{~Hz}, \mathrm{C} \underline{\mathrm{H}}(\mathrm{Ph})) ; 9.11(1 \mathrm{H}$, br. s, $\mathrm{N} \underline{\mathrm{H}}(\mathrm{Ind})) ;{ }^{13} C-N M R$ $\left(\mathrm{CDCl}_{3}\right) \delta=21.64\left(\underline{\mathrm{CH}}_{3}(\mathrm{i}-\mathrm{Pr})\right) ; 23.86\left(\underline{\mathrm{CH}}_{3}(\mathrm{i}-\mathrm{Pr})\right) ; 45.88\left(\underline{\mathrm{CH}}\left(\mathrm{i}-\mathrm{Pr}, \mathrm{J}_{\mathrm{C}-\mathrm{P}}=16.2 \mathrm{~Hz}\right) ; 49.93(\underline{\mathrm{CH}}-\right.$ $\left.\mathrm{P}, \mathrm{J}_{\mathrm{C}-\mathrm{P}}=163.8 \mathrm{~Hz}\right) ; 53.34\left(\underline{\mathrm{CH}}_{3} \mathrm{O}, \mathrm{J}_{\mathrm{C}-\mathrm{P}}=6.9 \mathrm{~Hz}\right) ; 53.87\left(\underline{\mathrm{CH}}_{3} \mathrm{O}, \mathrm{J}_{\mathrm{C}-\mathrm{P}}=5.8 \mathrm{~Hz}\right) ; 110.30(\underline{\mathrm{C}}-\mathrm{C}-\mathrm{N}$, $\left.\mathrm{J}_{\mathrm{C}-\mathrm{P}}=2.3 \mathrm{~Hz}\right) ; 111.66 ; 119.04 ; 119.66 ; 122.00(\underline{\mathrm{CH}}(\mathrm{Ph})) ; 124.29\left(\underline{\mathrm{CH}}-\mathrm{N}, \mathrm{J}_{\mathrm{C}-\mathrm{P}}=6.9 \mathrm{~Hz}\right) ; 126.89$ $\left(\underline{\mathrm{C}}_{\mathrm{q}}-\mathrm{CHP}, \mathrm{J}_{\mathrm{C}-\mathrm{P}}=5.8 \mathrm{~Hz}\right) ; 136.38\left(\underline{\mathrm{C}}_{\mathrm{q}}-\mathrm{N}\right) ; \mathrm{IR} 1036(\mathrm{P}-\mathrm{O}) ; 1227(\mathrm{P}=\mathrm{O}) ; 1457 ; 3244(\mathrm{NH}) ;{ }^{31} \mathrm{P}-$ $N M R\left(C D C l_{3}\right) \delta=28.13 ; M S 295\left(\mathrm{M}^{-}-1\right) ; H R M S$ Calc. for $\mathrm{C}_{14} \mathrm{H}_{21} \mathrm{~N}_{2} \mathrm{O}_{3} \mathrm{P}-\mathrm{H}^{+}: 295.1217$, found 295.1220; yellow oil.

Dimethyl cyclohexyl(isopropylamino)methylphosphonate (2l). ${ }^{1} \mathrm{H}-\mathrm{NMR}\left(\mathrm{CDCl}_{3}\right) \delta=0.99$ $\left(3 \mathrm{H}, \mathrm{d}, \mathrm{J}=6.3 \mathrm{~Hz}, \mathrm{C}_{3}(i-P r)\right) ; 1.04\left(3 \mathrm{H}, \mathrm{d}, \mathrm{J}=6.1 \mathrm{~Hz}, \underline{\mathrm{C}}_{3}(i-P r)\right) ; 1.07-1.46(6 \mathrm{H}, \mathrm{m}, \mathrm{CH}(c-$ Hex $\left.) ; 2 \mathrm{xCH}_{2}(c-\mathrm{Hex}) ; \mathrm{NH}\right) ; 1.61-1.87\left(6 \mathrm{H}, \mathrm{m}, 3 \mathrm{xCH}_{2}(c-\mathrm{Hex})\right) ; 2.78\left(1 \mathrm{H}, \mathrm{dd}, \mathrm{J}_{\mathrm{H}-\mathrm{P}}=16.8 \mathrm{~Hz}, \mathrm{~J}_{2}=\right.$ $3.3 \mathrm{~Hz}, \mathrm{C} \underline{\mathrm{H}}-\mathrm{P}) ; 2.98\left(1 \mathrm{H}\right.$, septet x d, J = 6.1 Hz, J $\left.\mathrm{H}_{-\mathrm{P}}=1.3 \mathrm{~Hz}, \mathrm{C} \underline{\mathrm{H}}(\mathrm{i}-\mathrm{Pr})\right) ; 3.75\left(3 \mathrm{H}, \mathrm{d}, \mathrm{J}_{\mathrm{H}-\mathrm{P}}=10.5\right.$ $\left.\mathrm{Hz}, \mathrm{CH}_{3} \mathrm{O}\right) ; 3.78\left(3 \mathrm{H}, \mathrm{d}, \mathrm{J}_{\mathrm{H}-\mathrm{P}}=10.5 \mathrm{~Hz}, \mathrm{CH}_{3} \mathrm{O}\right) ;{ }^{13} \mathrm{C}-\mathrm{NMR}\left(\mathrm{CDCl}_{3}\right) \delta=22.85\left(\underline{\mathrm{CH}}_{3}(\mathrm{i}-\mathrm{Pr})\right) ; 23.36$ $\left(\underline{\mathrm{CH}}_{3}(\mathrm{i}-\mathrm{Pr})\right) ; 26.19 ; 26.50 ; 26.68\left(3 \times \underline{\mathrm{CH}}_{2}(c-\mathrm{Hex})\right) ; 28.35\left(\underline{\mathrm{CH}}_{2}(c-\mathrm{Hex}), \mathrm{J}_{\mathrm{C}-\mathrm{P}}=3.5 \mathrm{~Hz}\right) ; 30.90$ $\left(\underline{\mathrm{CH}}_{2}(c-\mathrm{Hex}), \mathrm{J}_{\mathrm{C}-\mathrm{P}}=11.64 \mathrm{~Hz}\right) ; 39.62\left(\underline{\mathrm{CH}}(c-\mathrm{Hex}), \mathrm{J}_{\mathrm{C}-\mathrm{P}}=5.8 \mathrm{~Hz}\right) ; 47.72\left(\underline{\mathrm{CH}}(\mathrm{i}-\mathrm{Pr}), \mathrm{J}_{\mathrm{C}-\mathrm{P}}=5.77\right.$ $\mathrm{Hz}) ; 52.36\left(\underline{\mathrm{CH}}_{3} \mathrm{O}, \mathrm{J}_{\mathrm{C}-\mathrm{P}}=8.1 \mathrm{~Hz}\right) ; 52.87\left(\underline{\mathrm{CH}}_{3} \mathrm{O}, \mathrm{J}_{\mathrm{C}-\mathrm{P}}=8.1 \mathrm{~Hz}\right) ; 57.59\left(\underline{\mathrm{CH}}-\mathrm{P}, \mathrm{J}_{\mathrm{C}-\mathrm{P}}=144.2 \mathrm{~Hz}\right)$; IR 1030 (P-O), $1067(\mathrm{P}-\mathrm{O}), 1243(\mathrm{P}=\mathrm{O}), 3294 ; 3309 ; 3325(\mathrm{NH}) ;{ }^{1} \mathrm{P}-\mathrm{NMR}\left(\mathrm{CDCl}_{3}\right) \delta=31.77$; MS $264\left(\mathrm{M}^{+}+1\right)$; HRMS Calc. for $\mathrm{C}_{12} \mathrm{H}_{26} \mathrm{NO}_{3} \mathrm{P}+\mathrm{H}^{+}$: 264.1723, found 264.1723; mp 54.2 ${ }^{\circ} \mathrm{C}$; white solid.

Dimethyl 1-(isopropylamino)-2-methylpropylphosphonate (2m). ${ }^{1} \mathrm{H}-\mathrm{NMR}\left(\mathrm{CDCl}_{3}\right) \delta=0.98$ $1.06\left(12 \mathrm{H}, \mathrm{m}, 4 \mathrm{xCH}_{3}\right) ; 2.02(1 \mathrm{H}, \mathrm{m}, \mathrm{C} \underline{\mathrm{H}}-\mathrm{CHP}) ; 2.81\left(1 \mathrm{H}, \mathrm{dd}, \mathrm{J}_{\mathrm{H}-\mathrm{P}}=16.5 \mathrm{~Hz}, \mathrm{~J}_{2}=3.6 \mathrm{~Hz}, \mathrm{C} \underline{\mathrm{HP}}\right)$; $3.00\left(1 \mathrm{H}\right.$, dxseptet, $\left.\mathrm{J}=6.3 \mathrm{~Hz}, \mathrm{~J}_{\mathrm{H}-\mathrm{P}}=1.4 \mathrm{~Hz}, \mathrm{C} \underline{\mathrm{H}}(\mathrm{i}-\mathrm{Pr})\right) ; 3.75\left(3 \mathrm{H}, \mathrm{d}, \mathrm{J}_{\mathrm{H}-\mathrm{P}}=9.9 \mathrm{~Hz}, \mathrm{CH}_{3} \mathrm{O}\right) ; 3.79$ $\left(3 \mathrm{H}, \mathrm{d}, \mathrm{J}_{\mathrm{H}-\mathrm{P}}=10.2 \mathrm{~Hz}, \underline{\mathrm{C}}_{3} \mathrm{O}\right) ;{ }^{13} \mathrm{C}-\mathrm{NMR}\left(\mathrm{CDCl}_{3}\right) \delta=17.91\left(\mathrm{C}_{3}, \mathrm{~J}_{\mathrm{C}-\mathrm{P}}=2.3 \mathrm{~Hz}\right) ; 20.55\left(\underline{\mathrm{CH}}_{3}\right.$, $\left.\mathrm{J}_{\mathrm{C}-\mathrm{P}}=13.8 \mathrm{~Hz}\right) ; 22.72\left(\underline{\mathrm{CH}}_{3}(\mathrm{i}-\mathrm{Pr})\right) ; 23.45\left(\underline{\mathrm{CH}}_{3}(\mathrm{i}-\mathrm{Pr})\right) ; 29.26\left(\underline{\mathrm{CH}}-\mathrm{CHP}, \mathrm{J}_{\mathrm{C}-\mathrm{P}}=5.78 \mathrm{~Hz}\right) ; 47.51$ $\left(\underline{\mathrm{CH}}(\mathrm{i}-\mathrm{Pr}), \mathrm{J}_{\mathrm{C}-\mathrm{P}}=5.78 \mathrm{~Hz}\right) ; 52.30\left(\underline{\mathrm{CH}}_{3} \mathrm{O}, \mathrm{J}_{\mathrm{C}-\mathrm{P}}=8.1 \mathrm{~Hz}\right) ; 52.81\left(\underline{\mathrm{CH}}_{3} \mathrm{O}, \mathrm{J}_{\mathrm{C}-\mathrm{P}}=6.9 \mathrm{~Hz}\right) ; 57.39$ $\left(\underline{\mathrm{CHP}}, \mathrm{J}_{\mathrm{C}-\mathrm{P}}=144.2 \mathrm{~Hz}\right) ; \mathrm{IR} 1054(\mathrm{P}-\mathrm{O}) ; 1247(\mathrm{P}=\mathrm{O}) ; 1465 ; 3326(\mathrm{NH}) ;{ }^{31} \mathrm{P}-\mathrm{NMR}\left(\mathrm{CDCl}_{3}\right) \delta=$ 31.84; MS $114\left(\mathrm{M}^{+}-\mathrm{P}(\mathrm{O})(\mathrm{OMe})_{2}\right)$; HRMS Calc. for $\mathrm{C}_{9} \mathrm{H}_{22} \mathrm{NO}_{3} \mathrm{P}+\mathrm{H}^{+}$: 224.1410, found 224.1411; colourless oil.

Dimethyl 1-(tert-butylamino)-2-methylpropylphosphonate (2n). ${ }^{1} H-N M R\left(C D C l_{3}\right) \delta=1.01$ $\left(3 \mathrm{H}, \mathrm{d}, \mathrm{J}=7.2 \mathrm{~Hz}, \mathrm{CH}_{3} \mathrm{CH}\right) ; 1.03\left(3 \mathrm{H}, \mathrm{d}, \mathrm{J}=6.9 \mathrm{~Hz}, \mathrm{C}_{3} \mathrm{CH}\right) ; 1.09$ (9H, s, $\left.\mathrm{C}_{3}(t-b u)\right) ; 1.92-2.17$

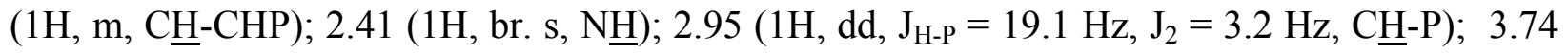
$\left(3 \mathrm{H}, \mathrm{d}, \mathrm{J}_{\mathrm{H}-\mathrm{P}}=10.5 \mathrm{~Hz}, \mathrm{CH}_{3} \mathrm{O}\right) ; 3.78\left(3 \mathrm{H}, \mathrm{d}, \mathrm{J}_{\mathrm{H}-\mathrm{P}}=10.5 \mathrm{~Hz}, \mathrm{CH}_{3} \mathrm{O}\right) ;{ }^{13} C-N M R\left(\mathrm{CDCl}_{3}\right) \delta=18.88$ $\left(\underline{\mathrm{CH}}_{3} \mathrm{CH}\right) ; 19.46\left(\underline{\mathrm{CH}}_{3} \mathrm{CH}, \mathrm{J}_{\mathrm{C}-\mathrm{P}}=11.5 \mathrm{~Hz}\right) ; 30.21\left(\underline{\mathrm{CH}}_{3}(t-\mathrm{bu})\right) ; 31.64\left(\underline{\mathrm{CH}}-\mathrm{CHP}, \mathrm{J}_{\mathrm{C}-\mathrm{P}}=6.9 \mathrm{~Hz}\right)$; $51.04\left(\underline{\mathrm{C}}_{\mathrm{q}}(t-\mathrm{bu}), \mathrm{J}_{\mathrm{C}-\mathrm{P}}=5.8 \mathrm{~Hz}\right) ; 53.23\left(\underline{\mathrm{CH}}_{3} \mathrm{O}, \mathrm{J}_{\mathrm{C}-\mathrm{P}}=8.1 \mathrm{~Hz}\right) ; 53.13\left(\underline{\mathrm{CH}}_{3} \mathrm{O}, \mathrm{J}_{\mathrm{C}-\mathrm{P}}=8.1 \mathrm{~Hz}\right) ; 54.62$ $\left(\underline{\mathrm{CH}}-\mathrm{P}, \mathrm{J}_{\mathrm{C}-\mathrm{P}}=147.68 \mathrm{~Hz}\right) ; \mathrm{IR} 1032(\mathrm{P}-\mathrm{O}) ; 1056(\mathrm{P}-\mathrm{O}) ; 1239(\mathrm{P}=\mathrm{O}) ; 1464 ; 3477(\mathrm{NH}) ;{ }^{31} \mathrm{P}-N M R$ 


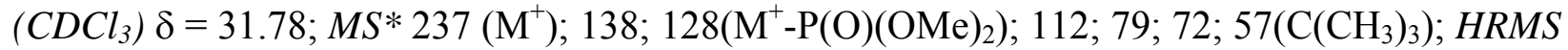
Calc. for $\mathrm{C}_{10} \mathrm{H}_{24} \mathrm{NO}_{3} \mathrm{P}+\mathrm{H}^{+}$: 238.1567, found 238.1564; colourless oil.

* In this case the mass spectrum was recorded using a Hewlett-Packard 6890 GC Plus coupled with a HP 5973 MSD (Mass Selective Detector-Quadrupole type), equipped with a CIS-4 PTV (Programmed Temperature Vaporisation) Injector (Gerstel).

Dimethyl 1-(benzylamino)-2-methylpropylphosphonate (2o). ${ }^{1} \mathrm{H}-\mathrm{NMR}\left(\mathrm{CDCl}_{3}\right) \delta=1.01(3 \mathrm{H}$, d, J = $\left.6.9 \mathrm{~Hz}, \mathrm{C}_{3}\right) ; 1.02\left(3 \mathrm{H}, \mathrm{dd}, \mathrm{J}=6.7 \mathrm{~Hz}, \mathrm{~J}_{\mathrm{H}-\mathrm{P}}=1.0 \mathrm{~Hz}, \underline{\mathrm{C}}_{3}\right) ; 1.50(1 \mathrm{H}, \mathrm{m}, \mathrm{N} \underline{\mathrm{H}}) ; 2.05-2.22$ $(1 \mathrm{H}, \mathrm{m}, \mathrm{CH}-\mathrm{CHP}) ; 2.78\left(1 \mathrm{H}, \mathrm{dd}, \mathrm{J}_{\mathrm{H}-\mathrm{P}}=14.5 \mathrm{~Hz}, \mathrm{~J}_{2}=3.7 \mathrm{~Hz}, \mathrm{C} \underline{\mathrm{H}}-\mathrm{P}\right) ; 3.76\left(3 \mathrm{H}, \mathrm{d}, \mathrm{J}_{\mathrm{H}-\mathrm{P}}=10.5 \mathrm{~Hz}\right.$, $\left.\mathrm{C}_{3} \mathrm{O}\right) ; 3.79\left(3 \mathrm{H}, \mathrm{d}, \mathrm{J}_{\mathrm{H}-\mathrm{P}}=10.2 \mathrm{~Hz}, \mathrm{C}_{3} \mathrm{O}\right) ; 3.83\left(1 \mathrm{H}, \mathrm{dd}, \mathrm{J}_{\mathrm{AB}}=12.6 \mathrm{~Hz}, \mathrm{~J}_{\mathrm{H}-\mathrm{P}}=1.4 \mathrm{~Hz}, \mathrm{C}_{2} \mathrm{~N}\right)$; $4.01\left(1 \mathrm{H}, \mathrm{d}, \mathrm{J}_{\mathrm{AB}}=12.6 \mathrm{~Hz}, \mathrm{C}_{2} \mathrm{Ph}\right) ; 7.22-7.38(5 \mathrm{H}, \mathrm{m}, \mathrm{C} \underline{\mathrm{H}}(\mathrm{Ph})) ;{ }^{13} C-N M R\left(C D C l_{3}\right) \delta=17.92$ $\left(\underline{\mathrm{CH}}_{3}, \mathrm{~J}_{\mathrm{C}-\mathrm{P}}=3.5 \mathrm{~Hz}\right) ; 20.63\left(\underline{\mathrm{CH}}_{3}, \mathrm{~J}_{\mathrm{C}-\mathrm{P}}=12.7 \mathrm{~Hz}\right) ; 29.03\left(\underline{\mathrm{CH}}-\mathrm{CHP}, \mathrm{J}_{\mathrm{C}-\mathrm{P}}=5.8 \mathrm{~Hz}\right) ; 52.35\left(\underline{\mathrm{CH}}_{3} \mathrm{O}\right.$, $\left.\mathrm{J}_{\mathrm{C}-\mathrm{P}}=6.9 \mathrm{~Hz}\right) ; 52.39\left(\underline{\mathrm{CH}}_{3} \mathrm{O}, \mathrm{J}_{\mathrm{C}-\mathrm{P}}=8.1 \mathrm{~Hz}\right) ; 53.26\left(\underline{\mathrm{CH}}_{2} \mathrm{~N}, \mathrm{~J}_{\mathrm{C}-\mathrm{P}}=3.5 \mathrm{~Hz}\right) ; 59.25\left(\underline{\mathrm{CHP}}, \mathrm{J}_{\mathrm{C}-\mathrm{P}}=\right.$ $142.0 \mathrm{~Hz}) ; 127.09 ; 128.27 ; 128.44(\underline{\mathrm{CH}}(\mathrm{Ph})) ; 140.08\left(\underline{\mathrm{C}}_{\mathrm{q}}(\mathrm{Ph})\right) ; \mathrm{IR} 1031$ (P-O); 1057 (P-O); 1246 $(\mathrm{P}=\mathrm{O}) ; 3469(\mathrm{NH}) ;{ }^{31} \mathrm{P}-\mathrm{NMR}\left(\mathrm{CDCl}_{3}\right) \delta=31.75 ; \mathrm{MS} 271\left(\mathrm{M}^{+}+1\right)$; HRMS Calc. for $\mathrm{C}_{13} \mathrm{H}_{22} \mathrm{NO}_{3} \mathrm{P}$ $+\mathrm{H}^{+}: 272.1410$, found: 274.1411; colourless oil.

Diethyl 1-(isopropylamino)-3-methylbut-2-enylphosphonate (2p). ${ }^{1} \mathrm{H}-\mathrm{NMR}\left(\mathrm{CDCl}_{3}\right) \delta=0.98$ $\left(3 \mathrm{H}, \mathrm{d}, \mathrm{J}=6.1 \mathrm{~Hz}, \underline{\mathrm{C}}_{3}(i-P r)\right) ; 1.05\left(3 \mathrm{H}, \mathrm{d}, \mathrm{J}=6.3 \mathrm{~Hz}, \mathrm{C}_{3}(i-P r)\right) ; 1.30(3 \mathrm{H}, \mathrm{t}, \mathrm{J}=7.2 \mathrm{~Hz}$, $\left.\mathrm{C}_{3} \mathrm{CH}_{2} \mathrm{O}\right) ; 1.32\left(3 \mathrm{H}, \mathrm{t}, \mathrm{J}=7.2 \mathrm{~Hz}, \mathrm{C}_{3} \mathrm{CH}_{2} \mathrm{O}\right) ; 1.70\left(3 \mathrm{H}, \mathrm{dd}, \mathrm{J}_{1}=1.4 \mathrm{~Hz}, \mathrm{~J}_{2}=3.6 \mathrm{~Hz}, \mathrm{C}_{3} \mathrm{Cq}\right)$; $1.78\left(3 \mathrm{H}, \mathrm{dd}, \mathrm{J}_{1}=1.4 \mathrm{~Hz}, \mathrm{~J}_{2}=5.0 \mathrm{~Hz}, \mathrm{CH}_{3} \mathrm{Cq}\right) ; 2.87(1 \mathrm{H}$, septet, J = $6.2 \mathrm{~Hz}, \mathrm{C} \underline{\mathrm{H}}(\mathrm{i}-\mathrm{Pr})) ; 3.80$ $\left(1 \mathrm{H}, \mathrm{dd}, \mathrm{J}_{\mathrm{H}-\mathrm{P}}=19.3 \mathrm{~Hz}, \mathrm{~J}_{2}=9.9 \mathrm{~Hz}, \mathrm{C} \underline{\mathrm{H}}-\mathrm{P}\right) ; 4.06-4.27\left(4 \mathrm{H}, \mathrm{m}, \mathrm{CH}_{3} \mathrm{C}_{2} \mathrm{O}\right) ; 5.00-5.07(1 \mathrm{H}, \mathrm{m}$, $=\mathrm{C} \underline{\mathrm{H}}) ;{ }^{13} \mathrm{C}-\mathrm{NMR}\left(\mathrm{CDCl}_{3}\right) \delta=16.53\left(\underline{\mathrm{C}}_{3} \mathrm{CH}_{2} \mathrm{O}\right) ; 16.59\left(\underline{\mathrm{CH}}_{3} \mathrm{CH}_{2} \mathrm{O}\right) ; 18.58\left(\underline{\mathrm{C}} \mathrm{H}_{3} \mathrm{Cq}\right) ; 21.97$ $\left(\underline{\mathrm{CH}}_{3}(\mathrm{i}-\mathrm{Pr})\right) ; 23.89\left(\underline{\mathrm{CH}}_{3}(i-\mathrm{Pr})\right) ; 26.01\left(\underline{\mathrm{CH}}_{3} \mathrm{Cq}, \mathrm{J}_{\mathrm{C}-\mathrm{P}}=2.31 \mathrm{~Hz}\right) ; 45.94\left(\mathrm{C} \underline{\mathrm{H}}(\mathrm{i}-\mathrm{Pr}), \mathrm{J}_{\mathrm{C}-\mathrm{P}}=16.2 \mathrm{~Hz}\right)$; $52.14\left(\underline{\mathrm{C}} \mathrm{H}-\mathrm{P}, \mathrm{J}_{\mathrm{C}-\mathrm{P}}=158.1 \mathrm{~Hz}\right) ; 62.43\left(\mathrm{CH}_{3} \underline{\mathrm{CH}}_{2} \mathrm{O}, \mathrm{J}_{\mathrm{C}-\mathrm{P}}=6.9 \mathrm{~Hz}\right) ; 62.72\left(\mathrm{CH}_{3} \underline{\mathrm{C}} \mathrm{H}_{2} \mathrm{O}, \mathrm{J}_{\mathrm{C}-\mathrm{P}}=8.1\right.$ $\mathrm{Hz}) ; 120.90\left(=\underline{\mathrm{CH}}, \mathrm{J}_{\mathrm{C}-\mathrm{P}}=4.62 \mathrm{~Hz}\right) ; 137.27\left(=\underline{\mathrm{Cq}}, \mathrm{J}_{\mathrm{C}-\mathrm{P}}=13.8 \mathrm{~Hz}\right) ; I R 1031(\mathrm{P}-\mathrm{O}) ; 1058(\mathrm{P}-\mathrm{O})$; $\left.1245(\mathrm{P}=\mathrm{O}) ; 3286(\mathrm{NH}) ;{ }^{31} \mathrm{P}-\mathrm{NMR}\left(\mathrm{CDCl}_{3}\right) \delta=26.42 ; M S 126\left(\mathrm{M}^{+}-\mathrm{P}(\mathrm{O})(\mathrm{OEt})_{2}\right)\right)$; HRMS Calc. for $\mathrm{C}_{10} \mathrm{H}_{22} \mathrm{NO}_{3} \mathrm{P}+\mathrm{H}^{+}: 236.1410$, found: 236.1412; colourless oil.

Dimethyl (2E)-1-(allylamino)-3-phenylprop-2-enylphosphonate (2q). ${ }^{1} \mathrm{H}-\mathrm{NMR}\left(\mathrm{CDCl}_{3}\right) \delta=$ $1.69(1 \mathrm{H}$, br. s, $\mathrm{N} \underline{\mathrm{H}}) ; 3.21\left(1 \mathrm{H}, \mathrm{dd}, \mathrm{J}_{\mathrm{AB}}=14.0 \mathrm{~Hz}, \mathrm{~J}_{2}=6.3 \mathrm{~Hz}, \mathrm{C}_{2} \mathrm{~N}\right) ; 3.41\left(1 \mathrm{H}, \mathrm{dd}, \mathrm{J}_{\mathrm{AB}}=14.0\right.$ $\left.\mathrm{Hz}, \mathrm{J}_{2}=5.2 \mathrm{~Hz}, \underline{\mathrm{C}}_{2} \mathrm{~N}\right) ; 3.72-3.84\left(7 \mathrm{H}, \mathrm{m}, \mathrm{C} \underline{\mathrm{HP}}, \underline{\mathrm{C}}_{3} \mathrm{O}\right) ; 5.12-5.22\left(2 \mathrm{H}, \mathrm{m},=\mathrm{C}_{2}\right) ; 5.79-5.90$ $\left(1 \mathrm{H}\right.$, dddd, $\left.\mathrm{J}_{1}=17.2 \mathrm{~Hz}, \mathrm{~J}_{2}=10.3 \mathrm{~Hz}, \mathrm{~J}_{3}=6.7 \mathrm{~Hz}, \mathrm{~J}_{4}=5.2 \mathrm{~Hz}, \mathrm{CH}_{2}=\mathrm{CH}\right) ; 6.10\left(1 \mathrm{H}, \mathrm{ddd}, \mathrm{J}_{\mathrm{AB}}=\right.$ $\left.15.8 \mathrm{~Hz}, \mathrm{~J}_{2}=8.7 \mathrm{~Hz}, \mathrm{~J}_{\mathrm{H}-\mathrm{P}}=5.8 \mathrm{~Hz}, \mathrm{Ph}-\mathrm{CH}=\mathrm{CH}\right) ; 6.62\left(1 \mathrm{H}, \mathrm{dd}, \mathrm{J}_{\mathrm{AB}}=16.0 \mathrm{~Hz}, \mathrm{~J}_{2}=4.7 \mathrm{~Hz}\right.$, $\mathrm{PhC} \underline{\mathrm{H}}=\mathrm{CH}) ; 7.23-7.42(5 \mathrm{H}, \mathrm{m}, \mathrm{C} \underline{\mathrm{H}}(\mathrm{Ph})) ;{ }^{13} \mathrm{C}-\mathrm{NMR}\left(\mathrm{CDCl}_{3}\right) \delta=50.09\left(\underline{\mathrm{CH}}_{2} \mathrm{~N}, \mathrm{~J}_{\mathrm{C}-\mathrm{P}}=16.2 \mathrm{~Hz}\right)$; $53.57\left(\underline{\mathrm{CH}}_{3} \mathrm{O}, \mathrm{J}_{\mathrm{C}-\mathrm{P}}=6.9 \mathrm{~Hz}\right) ; 53.73\left(\underline{\mathrm{CH}}_{3} \mathrm{O}, \mathrm{J}_{\mathrm{C}-\mathrm{P}}=8.1 \mathrm{~Hz}\right) ; 57.72\left(\underline{\mathrm{CHP}}, \mathrm{J}_{\mathrm{C}-\mathrm{P}}=156.91 \mathrm{~Hz}\right)$; $116.96\left(=\underline{\mathrm{CH}}_{2}\right) ; 123.91\left(\mathrm{PhCH}=\underline{\mathrm{C} H}, \mathrm{~J}_{\mathrm{C}-\mathrm{P}}=6.9 \mathrm{~Hz}\right) ; 126.64 ; 128.09 ; 128.72(\underline{\mathrm{CH}}(\mathrm{Ph})) ; 134.70$ $\left(\mathrm{PhCH}=\mathrm{CH}, \mathrm{J}_{\mathrm{C}-\mathrm{P}}=6.9 \mathrm{~Hz}\right) ; 135.97\left(\underline{\mathrm{CH}}=\mathrm{CH}_{2}\right) ; 136.34\left(\mathrm{C}_{\mathrm{q}}(\mathrm{Ph})\right) ; \mathrm{IR} 1033(\mathrm{P}-\mathrm{O}) ; 1053(\mathrm{P}-\mathrm{O})$; $1247(\mathrm{P}=\mathrm{O}) ; 3308(\mathrm{NH}) ;{ }^{31} \mathrm{P}-\mathrm{NMR}\left(\mathrm{CDCl}_{3}\right) \delta=26.77$; MS $282\left(\mathrm{M}^{+}+1\right)$; HRMS Calc. for $\mathrm{C}_{14} \mathrm{H}_{20} \mathrm{NO}_{3} \mathrm{P}+\mathrm{H}^{+}: 282.1254$, found: 282,$1258 ; m p 54.1^{\circ} \mathrm{C}$; yellow solid.

Dimethyl (2E)-1-(tert-butylamino)-3-phenylprop-2-enylphosphonate(2r). ${ }^{1} \mathrm{H}-\mathrm{NMR}\left(\mathrm{CDCl}_{3}\right) \delta$ $=1.12\left(9 \mathrm{H}, \mathrm{s}, \mathrm{C}_{3}(t-b u)\right) ; 1.40(1 \mathrm{H}$, br. s, $\mathrm{N} \underline{\mathrm{H}}) ; 3.76\left(3 \mathrm{H}, \mathrm{d}, \mathrm{J}_{\mathrm{H}-\mathrm{P}}=10.5 \mathrm{~Hz}, \underline{\mathrm{C}}_{3} \mathrm{O}\right) ; 3.84(3 \mathrm{H}, \mathrm{d}$, $\left.\mathrm{J}_{\mathrm{H}-\mathrm{P}}=10.5 \mathrm{~Hz}, \underline{\mathrm{CH}}_{3} \mathrm{O}\right) ; 3.90\left(1 \mathrm{H}, \mathrm{dd}, \mathrm{J}_{\mathrm{H}-\mathrm{P}}=24.2 \mathrm{~Hz}, \mathrm{~J}_{2}=8.0 \mathrm{~Hz}, \mathrm{C} \underline{\mathrm{HP}}\right) ; 6.21\left(1 \mathrm{H}, \mathrm{ddd}, \mathrm{J}_{\mathrm{AB}}=\right.$ 
$\left.16.0 \mathrm{~Hz}, \mathrm{~J}_{2}=8.0 \mathrm{~Hz}, \mathrm{~J}_{\mathrm{H}-\mathrm{P}}=5.8 \mathrm{~Hz}, \mathrm{PhCH}=\mathrm{CH}\right) ; 6.63\left(1 \mathrm{H}, \mathrm{dd}, \mathrm{J}_{\mathrm{AB}}=16.0 \mathrm{~Hz}, \mathrm{~J}_{2}=5.2 \mathrm{~Hz}\right.$, $=\mathrm{C} \underline{\mathrm{HPh}}) ; 7.21-7.44(5 \mathrm{H}, \mathrm{m}, \mathrm{C} \underline{\mathrm{H}}(\mathrm{Ph})) ;{ }^{13} \mathrm{C}-N M R\left(\mathrm{CDCl}_{3}\right) \delta=30.00\left(\underline{\mathrm{CH}}_{3}(t-\mathrm{bu}) ; 52.18\left(\mathrm{C}_{\mathrm{q}}(t-\mathrm{bu})\right.\right.$, $\left.\mathrm{J}_{\mathrm{C}-\mathrm{P}}=15.0 \mathrm{~Hz}\right) ; 53.29\left(\underline{\mathrm{CH}}_{3} \mathrm{O}, \mathrm{J}_{\mathrm{C}-\mathrm{P}}=6.9 \mathrm{~Hz}\right) ; 53.89\left(\underline{\mathrm{CHP}}, \mathrm{J}_{\mathrm{C}-\mathrm{P}}=158.1 \mathrm{~Hz}\right) ; 54.43\left(\underline{\mathrm{CH}}_{3} \mathrm{O}, \mathrm{J}_{\mathrm{C}-\mathrm{P}}=\right.$ $6.9 \mathrm{~Hz}) ; 126.45\left(\underline{\mathrm{CH}}(\mathrm{Ph}), \mathrm{J}_{\mathrm{C}-\mathrm{P}}=2.3 \mathrm{~Hz}\right) ; 127.70(\underline{\mathrm{CH}}(\mathrm{Ph})) ; 127.96\left(\mathrm{PhCH}=\underline{\mathrm{CH}}, \mathrm{J}_{\mathrm{C}-\mathrm{P}}=4.6 \mathrm{~Hz}\right)$; $128.59(\underline{\mathrm{CH}}(\mathrm{Ph})) ; 132.30\left(\mathrm{Ph} \underline{\mathrm{CH}}=\mathrm{CH}, \mathrm{J}_{\mathrm{C}-\mathrm{P}}=13.8 \mathrm{~Hz}\right) ; 136.64\left(\mathrm{C}_{\mathrm{q}}(\mathrm{Ph})\right) ; \mathrm{IR} 1030(\mathrm{P}-\mathrm{O}) ; 1060(\mathrm{P}-$ $\mathrm{O}) ; 1240(\mathrm{P}=\mathrm{O}) ; 3298(\mathrm{NH}) ;{ }^{31} \mathrm{P}-\mathrm{NMR}\left(\mathrm{CDCl}_{3}\right) \delta=27.02 ; \mathrm{MS} 298\left(\mathrm{M}^{+}+1\right), 188\left(\mathrm{M}^{+}-\right.$ $\left.\mathrm{P}(\mathrm{O})(\mathrm{OMe})_{2}\right)$; HRMS Calc. for $\mathrm{C}_{15} \mathrm{H}_{24} \mathrm{NO}_{3} \mathrm{P}+\mathrm{H}^{+}$: 298.1567, found 298.1568; mp $54.7{ }^{\circ} \mathrm{C}$; yellow solid.

Dimethyl (2E)-1-(isopropylamino)-3-phenylprop-2-enylphosphonate (2s). ${ }^{1} \mathrm{H}-\mathrm{NMR}\left(\mathrm{CDCl}_{3}\right)$ $\delta=1.03\left(3 \mathrm{H}, \mathrm{d}, \mathrm{J}=6.3 \mathrm{~Hz}, \underline{\mathrm{H}}_{3}(\mathrm{i}-\mathrm{Pr})\right) ; 1.09\left(3 \mathrm{H}, \mathrm{d}, \mathrm{J}=6.3 \mathrm{~Hz}, \underline{\mathrm{C}}_{3}(\mathrm{i}-\mathrm{Pr})\right) ; 1.53(1 \mathrm{H}, \mathrm{br} . \mathrm{s}, \mathrm{N} \underline{\mathrm{H}})$; $2.95(1 \mathrm{H}$, septet, $\mathrm{J}=6.3 \mathrm{~Hz}, \mathrm{C} \underline{\mathrm{H}}(\mathrm{i}-\mathrm{Pr})) ; 3.78\left(3 \mathrm{H}, \mathrm{d}, \mathrm{J}_{\mathrm{H}-\mathrm{P}}=10.5 \mathrm{~Hz}, \mathrm{CH}_{3} \mathrm{O}\right) ; 3.83\left(3 \mathrm{H}, \mathrm{d}, \mathrm{J}_{\mathrm{H}-\mathrm{P}}=\right.$ $\left.10.5 \mathrm{~Hz}, \mathrm{C}_{3} \mathrm{O}\right) ; 3.82\left(1 \mathrm{H}, \mathrm{ddd}, \mathrm{J}_{\mathrm{H}-\mathrm{P}} \sim 20 \mathrm{~Hz}, \mathrm{~J}_{2}=8.5 \mathrm{~Hz}, \mathrm{~J}_{3}=1.1 \mathrm{~Hz}, \mathrm{C} \underline{\mathrm{HP}}\right) ; 6.12\left(1 \mathrm{H}, \mathrm{ddd}, \mathrm{J}_{\mathrm{AB}}\right.$ $\left.=16.0 \mathrm{~Hz}, \mathrm{~J}_{2}=8.5 \mathrm{~Hz}, \mathrm{~J}_{\mathrm{H}-\mathrm{P}}=5.8 \mathrm{~Hz}, \mathrm{PhCH}=\mathrm{CH}\right) ; 6.61\left(1 \mathrm{H}, \mathrm{dd}, \mathrm{J}_{\mathrm{AB}}=16.0 \mathrm{~Hz}, \mathrm{~J}_{\mathrm{H}-\mathrm{P}}=4.7 \mathrm{~Hz}\right.$, $=\mathrm{C} \underline{\mathrm{HPh}}) ; 7.23-7.42(5 \mathrm{H}, \mathrm{m}, \mathrm{C} \underline{\mathrm{H}}(\mathrm{Ph})) ;{ }^{13} \mathrm{C}-\mathrm{NMR}\left(\mathrm{CDCl}_{3}\right) \delta=21.56\left(\underline{\mathrm{CH}}_{3}(\mathrm{i}-\mathrm{Pr})\right) ; 23.93\left(\underline{\mathrm{CH}}_{3}(i-\right.$ $\operatorname{Pr})) ; 46.05\left(\underline{\mathrm{CH}}(\mathrm{i}-\mathrm{Pr}), \mathrm{J}_{\mathrm{C}-\mathrm{P}}=16.2 \mathrm{~Hz}\right) ; 53.44\left(\underline{\mathrm{CH}}_{3} \mathrm{O}, \mathrm{J}_{\mathrm{C}-\mathrm{P}}=6.9 \mathrm{~Hz}\right) ; 53.89\left(\underline{\mathrm{CH}}_{3} \mathrm{O}, \mathrm{J}_{\mathrm{C}-\mathrm{P}}=8.1\right.$ $\mathrm{Hz}) ; 56.26\left(\underline{\mathrm{CHP}}, \mathrm{J}_{\mathrm{C}-\mathrm{P}}=156.9 \mathrm{~Hz}\right) ; 124.69\left(\mathrm{PhCH}=\underline{\mathrm{C}} \mathrm{H}, \mathrm{J}_{\mathrm{C}-\mathrm{P}}=5.8 \mathrm{~Hz}\right) ; 126.56 ; 127.92 ; 128.60$ $(\underline{\mathrm{C}} \mathrm{H}(\mathrm{Ph})) ; 133.84\left(\mathrm{Ph} \underline{\mathrm{CH}}=\mathrm{CH}, \mathrm{J}_{\mathrm{C}-\mathrm{P}}=13.9 \mathrm{~Hz}\right) ; 136.31\left(\mathrm{C}_{\mathrm{q}}(\mathrm{Ph})\right) ; \mathrm{IR} 1033(\mathrm{P}-\mathrm{O}) ; 1061(\mathrm{P}-\mathrm{O})$; $1242(\mathrm{P}=\mathrm{O}) ; 3316(\mathrm{NH}) ;{ }^{31} \mathrm{P}-\mathrm{NMR}\left(\mathrm{CDCl}_{3}\right) \delta=27.04 ;$ MS $174\left(\mathrm{M}^{+}-\mathrm{P}(\mathrm{O})(\mathrm{OMe})_{2}\right)$; HRMS Calc. for $\mathrm{C}_{14} \mathrm{H}_{22} \mathrm{NO}_{3} \mathrm{P}+\mathrm{H}^{+}: 284.1410$, Found: $284.1408 ; m p 67.3{ }^{\circ} \mathrm{C}$; yellow solid.

Diethyl (2E)-1-(isopropylamino)-3-phenylprop-2-enylphosphonate (2t). ${ }^{1} \mathrm{H}-\mathrm{NMR}\left(\mathrm{CDCl}_{3}\right) \delta$ $=1.02\left(3 \mathrm{H}, \mathrm{d}, \mathrm{J}=6.1 \mathrm{~Hz}, \underline{\mathrm{C}}_{3}(i-P r)\right) ; 1.09\left(3 \mathrm{H}, \mathrm{d}, \mathrm{J}=6.3 \mathrm{~Hz}, \mathrm{C}_{3}(i-P r)\right) ; 1.31(3 \mathrm{H}, \mathrm{t}, \mathrm{J}=7.3$ $\left.\mathrm{Hz}, \underline{\mathrm{C}}_{3} \mathrm{CH}_{2} \mathrm{O}\right) ; 1.33\left(3 \mathrm{H}, \mathrm{t}, \mathrm{J}=7.3 \mathrm{~Hz}, \mathrm{C}_{3} \mathrm{CH}_{2} \mathrm{O}\right) ; 1.60(1 \mathrm{H}$, br. s, $\mathrm{N} \underline{\mathrm{H}}) ; 2.95$ (1H, septet, $\mathrm{J}=$ $6.2 \mathrm{~Hz}, \mathrm{C} \underline{\mathrm{H}}(\mathrm{i}-\mathrm{Pr})) ; 3.77\left(1 \mathrm{H}, \mathrm{dd}, \mathrm{J}_{\mathrm{H}-\mathrm{P}}=21.2 \mathrm{~Hz}, \mathrm{~J}_{2}=8.5 \mathrm{~Hz}, \mathrm{C} \underline{\mathrm{HP}}\right) ; 4.09-4.26(4 \mathrm{H}, \mathrm{m}$, $\left.\mathrm{CH}_{3} \underline{\mathrm{C}}_{2} \mathrm{O}\right) ; 6.12\left(1 \mathrm{H}, \mathrm{ddd}, \mathrm{J}_{\mathrm{AB}}=16.0 \mathrm{~Hz}, \mathrm{~J}_{2}=8.5 \mathrm{~Hz}, \mathrm{~J}_{\mathrm{H}-\mathrm{P}}=5.6 \mathrm{~Hz}, \mathrm{PhCH}=\mathrm{C} \underline{\mathrm{H}}\right) ; 6.60(1 \mathrm{H}, \mathrm{dd}$, $\left.\mathrm{J}_{\mathrm{AB}}=16.0 \mathrm{~Hz}, \mathrm{~J}_{2}=4.7 \mathrm{~Hz},=\mathrm{C} \underline{\mathrm{HPh}}\right) ; 7.23-7.41(5 \mathrm{H}, \mathrm{m}, \mathrm{C} \underline{\mathrm{H}}(\mathrm{Ph})) ;{ }^{13} \mathrm{C}-\mathrm{NMR}\left(\mathrm{CDCl}_{3}\right) \delta=16.58$ $\left(\underline{\mathrm{CH}}_{3} \mathrm{CH}_{2} \mathrm{O}\right) ; 16.62\left(\underline{\mathrm{CH}}_{3} \mathrm{CH}_{2} \mathrm{O}\right) ; 21.72\left(\underline{\mathrm{CH}}_{3}(\mathrm{i}-\mathrm{Pr})\right) ; 24.01\left(\underline{\mathrm{CH}}_{3}(\mathrm{i}-\mathrm{Pr})\right) ; 46.23\left(\underline{\mathrm{C}} \mathrm{H}(\mathrm{i}-\mathrm{Pr}), \mathrm{J}_{\mathrm{C}-\mathrm{P}}=\right.$ $16.2 \mathrm{~Hz}) ; 56.71\left(\underline{\mathrm{CHP}}, \mathrm{J}_{\mathrm{C}-\mathrm{P}}=155.8 \mathrm{~Hz}\right) ; 62.76\left(\mathrm{CH}_{3} \underline{\mathrm{CH}}_{2} \mathrm{O}, \mathrm{J}_{\mathrm{C}-\mathrm{P}}=6.9 \mathrm{~Hz}\right) ; 63.14\left(\mathrm{CH}_{3} \underline{\mathrm{CH}_{2}} \mathrm{O}_{\mathrm{C}-\mathrm{P}}\right.$ $=6.9 \mathrm{~Hz}) ; 125.27\left(\mathrm{PhCH}=\underline{\mathrm{CH}}, \mathrm{J}_{\mathrm{C}-\mathrm{P}}=6.9 \mathrm{~Hz}\right) ; 126.57 ; 127.86 ; 128.67(\underline{\mathrm{CH}}(\mathrm{Ph})) ; 133.62$ $\left(\mathrm{PhCH}=\mathrm{CH}, \mathrm{J}_{\mathrm{C}-\mathrm{P}}=13.9 \mathrm{~Hz}\right) ; 136.60\left(\mathrm{C}_{\mathrm{q}}(\mathrm{Ph})\right) ; \mathrm{IR} 1028(\mathrm{P}-\mathrm{O}) ; 1056(\mathrm{P}-\mathrm{O}) ; 1239(\mathrm{P}=\mathrm{O}) ; 3307$ $(\mathrm{NH}) ;{ }^{31} \mathrm{P}-\mathrm{NMR}\left(\mathrm{CDCl}_{3}\right) \delta=24.85$; MS $312\left(\mathrm{M}^{+}+1\right)$; HRMS Calc. for $\mathrm{C}_{16} \mathrm{H}_{26} \mathrm{NO}_{3} \mathrm{P}+\mathrm{H}^{+}$: 312.1723, found 312.1724 ; yellow oil.

Diethyl (2E)-1-(benzylamino)-3-phenylprop-2-enylphosphonate (2u) $\cdot{ }^{1} \mathrm{H}-\mathrm{NMR}\left(\mathrm{CDCl}_{3}\right) \delta=$ $1.29\left(3 \mathrm{H}, \mathrm{t}, \mathrm{J}=6.9 \mathrm{~Hz}, \mathrm{C}_{3} \mathrm{CH}_{2} \mathrm{O}\right) ; 1.31\left(3 \mathrm{H}, \mathrm{t}, \mathrm{J}=6.9 \mathrm{~Hz}, \underline{\mathrm{C}}_{3} \mathrm{CH}_{2} \mathrm{O}\right) ; 2.28(1 \mathrm{H}$, br. s, $\mathrm{N} \underline{\mathrm{H}})$; $3.67\left(1 \mathrm{H}, \mathrm{ddd}, \mathrm{J}_{\mathrm{H}-\mathrm{P}}=19.3 \mathrm{~Hz}, \mathrm{~J}_{2}=8.5 \mathrm{~Hz}, \mathrm{~J}_{3}=0.8 \mathrm{~Hz}, \mathrm{C} \underline{\mathrm{HP}}\right) ; 3.75\left(1 \mathrm{H}, \mathrm{d}, \mathrm{J}_{\mathrm{AB}}=13.6 \mathrm{~Hz}\right.$, $\left.\mathrm{C}_{2} \mathrm{Ph}\right) ; 3.97\left(1 \mathrm{H}, \mathrm{d}, \mathrm{J}_{\mathrm{AB}}=13.6 \mathrm{~Hz}, \underline{\mathrm{CH}}_{2} \mathrm{Ph}\right) ; 4.06-4.25\left(4 \mathrm{H}, \mathrm{m}, \mathrm{CH}_{3} \underline{\mathrm{C}}_{2} \mathrm{O}\right) ; 6.15\left(1 \mathrm{H}\right.$, ddd, $\mathrm{J}_{\mathrm{AB}}$ $\left.=16.0 \mathrm{~Hz}, \mathrm{~J}_{2}=8.5 \mathrm{~Hz}, \mathrm{~J}_{\mathrm{H}-\mathrm{P}}=5.8 \mathrm{~Hz}, \mathrm{PhCH}=\mathrm{CH}\right) ; 6.61\left(1 \mathrm{H}, \mathrm{dd}, \mathrm{J}_{\mathrm{AB}}=16.0 \mathrm{~Hz}, \mathrm{~J}_{2}=4.7 \mathrm{~Hz}\right.$, $=\mathrm{C} \underline{H P h}) ; 7.22-7.42(5 \mathrm{H}, \mathrm{m}, \mathrm{C} \underline{\mathrm{H}}(\mathrm{Ph})) ;{ }^{13} \mathrm{C}-\mathrm{NMR}\left(\mathrm{CDCl}_{3}\right) \delta=16.28 ; 16.36 ; 16.46 ; 16.54$ $\left(\underline{\mathrm{CH}}_{3} \mathrm{CH}_{2} \mathrm{O}\right) ; 51.27\left(\underline{\mathrm{CH}}_{2}, \mathrm{~J}_{\mathrm{C}-\mathrm{P}}=16.2 \mathrm{~Hz}\right) ; 57.72\left(\underline{\mathrm{CHP}}, \mathrm{J}_{\mathrm{C}-\mathrm{P}}=154.6 \mathrm{~Hz}\right) ; 62.71 ; 62.82 ; 62.86$; $62.95\left(\mathrm{CH}_{3} \underline{\mathrm{C}} \mathrm{H}_{2} \mathrm{O}\right) ; 124.19\left(\mathrm{PhCH}=\underline{\mathrm{CH}}, \mathrm{J}_{\mathrm{C}-\mathrm{P}}=6.9 \mathrm{~Hz}\right) ; 126.55 ; 127.13 ; 127.90 ; 128.30 ; 128.42$; $128.62(\underline{\mathrm{CH}}(\mathrm{Ph})) ; 133.49\left(\mathrm{Ph} \underline{\mathrm{CH}}=\mathrm{CH}, \mathrm{J}_{\mathrm{C}-\mathrm{P}}=13.8 \mathrm{~Hz}\right) ; 136.41\left(\mathrm{C}_{\mathrm{q}}(\mathrm{Ph}) \mathrm{CH}=\mathrm{CH}, \mathrm{J}_{\mathrm{C}-\mathrm{P}}=2.3 \mathrm{~Hz}\right)$; 
$139.37\left(\mathrm{CH}_{2} \mathrm{C}_{\mathrm{q}}(\mathrm{Ph})\right)$; IR $1028(\mathrm{P}-\mathrm{O}) ; 1050(\mathrm{P}-\mathrm{O}) ; 1243(\mathrm{P}=\mathrm{O}) ; 3305(\mathrm{NH}) ;{ }^{31} \mathrm{P}-\mathrm{NMR}\left(C D C l_{3}\right) \delta=$ 24.42; MS $360\left(\mathrm{M}^{+}+1\right), 222\left(\mathrm{M}^{+}-\mathrm{P}(\mathrm{O})(\mathrm{OEt})_{2}\right)$; HRMS Calc. for $\mathrm{C}_{20} \mathrm{H}_{26} \mathrm{NO}_{3} \mathrm{P}+\mathrm{H}^{+}: 360.1723$, found: 360.1721 ; yellow oil.

\section{Acknowledgements}

We are grateful to the Fund for Scientific Research Flanders (FWO-Vlaanderen) and Ghent University (BOF) for financial assistance. We also thank CPC-Cellular Process Chemistry Systems $\mathrm{GmbH}$ for their assistance and for valuable discussions. ${ }^{9}$

\section{References and Footnotes}

1. Moonen, K; Laureyn, I.; Stevens, C.V. Chem. Rev. 2004, 104, 6177.

2. Laureyn, I.; Stevens, C.V.; Soroka, M.; Malyse, P. Arkivoc 2003, (iv), 102.

3. Kafarski, P.; Lejczak, B. Phosphorus, Sulfur Silicon Rel. 1991, 63, 193.

4. Allen, M. C.; Fuhrer, W.; Tuck, B.; Wade, R.; Wood, J. M. J. Med. Chem. 1989, 32, 1652.

5. Atherton, F. R.; Hassal, C. H.1; Lambert, R. W. J. Med. Chem. 1986, 29, 29.

6. Taghavi-Moghadam, S.; Kleemann, A.; Golbig, K.G. Org. Process Res. Developm. 2001, 5, 652.

7. Zhang, X.; Stefanick, S.; Villani, F.J. Org. Process Res. Developm. 2004, 8, 455.

8. Panke, G.; Schwalbe, T.; Stirner, W.; Taghavi-Moghadam, S.; Wille, G. Synthesis 2003, 2827.

9. CPC - Cellular Process Chemistry Systems GmbH: Heiligkreuzweg 90, D-55130 Mainz, Germany, www.cpc-net.com. Schwalbe, T.; Golbig, K.; Hohmann, M.; Georg, P.; Oberbeck, A.; Dittmann, B.; Stasna, J.; Oberbeck, S. (Cellular Process Chemistry Inc., USA) Eur. Pat. Appl. 2001, EP 1123 734; Chem. Abstr. 2001, 135, 154468b.

10. Cherkasov, R. A.; Galkin, V. I. Russ. Chem. Rev. 1998, 67, 857.

11. Gancarz, R. Tetrahedron 1995, 51, 10627.

12. Chandrasekhar, S.; Narsihmulu, Ch.; Shameem Sultana, S.; Saritha, B.; Jaya Prakash, S. Synlett 2003, 505.

13. Takahashi, H.; Yoshioka, M.; Imai, N.; Onimura, K.; Kobayashi, S. Synthesis 1994, 763.

14. Heydari, A.; Karimian,A.; Ipaktschi, J. Tetrahedron Lett. 1998, 39, 6729.

15. Azizi, N.; Saidi, M. R. Eur. J. Org. Chem. 2003, 4630.

16. Chandrasekhar, S.; Prakash, S. J.; Jagadeshwar; V., Narsihmulu, Ch. Tetrahedron Lett. 2001, 42, 5561.

17. Ranu, B. C.; Hajra, A.; Jana, U. Org. Lett. 1999, 1, 1141.

18. Manabe, K.; Kobayashi, S. Chem. Commun. 2000, 669.

19. Kaboudin, B.; Rahmani, A. Synthesis 2003, 2705. 
20. Lee, S.; Park, J. H.; Kang, J.; Lee, J. K. Chem. Commun. 2001, 1698.

21. Akiyama, T.; Sanada, M.; Fuchibe, K. Synlett 2003, 1463.

22. Henderson, W. M.; Shelver, W. H. J. Pharm. Sci. 1969, 58, 106.

23. Hopkins, L .V.; Vacik, J. P.; Shelver, W. H. J. Pharm. Sci. 1972, 61, 114.

24. Oussaid, A.; Benyaqad, F.; Oussaid, B.; Pradel, C.; Garrigues, B. Phosphorous, Sulfur, Silicon 2003, 178, 1183.

25. Yager, K. M.; Taylor, C. M.; Smith, A. B. J. Am. Chem. Soc. 1994, 116, 9377.

26. Simoni, D.; Invidiata, F. P.; Manferdini, M.; Lampronti, I.; Rondanin, R.; Roberti, M.; Pollini, G. P. Tetrahedron Lett. 1998, 39, 7615.

27. Klepacz, A.; Zwierzak, A. Tetrahedron Lett. 2002, 43, 1079.

28. Manjula, A.; Rao, V.; Neelakantan, P. Synth. Commun. 2003, 33, 2963.

29. Doye, S. Synlett 2004, 1653.

30. Schlemminger, I.; Willecke, A.; Maison, W.; Koch, R.; Lützen, A.; Martens, J. J. Chem. Soc. Perkin Trans. 1 2001, 2804.

31. Laschat S.; Kunz H. Synthesis 1992, 90.

32. Yadav, J. S.; Reddy, B. V. S.; Raj, K. S.; Reddy, B.; Prasad, A. R. Synthesis 2001, 2277.

33. Afarinkia, K.; Rees, C. W.; Cadogan, J. I. G. Tetrahedron 1990, 46, 7175.

34. Afarinkia, K.; Cadogan, J. I. G.; Rees, C. W. Synlett 1992, 123.

35. Bongini, A.; Camerini, R.; Panunzio, M. Tetrahedron: Asymmetry 1996, 7, 1467.

36. Rassukana, Y.V.; Onys'ko, P.; Grechukha, G.; Sinitsa, A. D. Eur. J. Org. Chem. 2003, 4181.

37. Boduszek, B. Pol. J. Chem. 2001, 75, 663.

38. Boduszek, B.; Soroka, M. Pol. J. Chem. 2002, 76, 1105.

39. Teulade, M.-P.; Savignac, P. Synthesis 1987, 1037.

40. Jahn, U.; Andersch, J.; Schroth, W. Synthesis 1997, 573.

41. Atmani, A.; Combret, J.-C.; Malhiac, C.; Mulengi, J. K. Tetrahedron Lett. 2000, 41, 6045.

42. Moonen, K.; Stevens, C.V. Synthesis, 2005, in press.

43. Fields, E. K. J. Am. Chem. Soc. 1952, 74, 1528.

44. Tyka, R. Tetrahedron Lett. 1970, 677.

45. Sobanov, A. A.; Zolotukhin, A. V.; Galkin, V. I.; Cherkasov, R. A.; Pudovik, A. N. Russ. J. Gen. Chem. 2002, 72, 1067.

46. Cristau, H.-J.; Coulombeau, A.; Genevois-Borella, A.; Pirat, J.-L. Tetrahedron Lett. 2001, 42, 4491.

47. Lewkowski, J.; Rzezniczak, M.; Skowronski, R. Org. Prep. Proced. Int. 2000, 32, 453. 